\title{
Faut-il rompre avec les quasi-marchés pour penser la marchandisation?
}

Réflexion autour du cas de l'aide à domicile

\section{Sylvain Vatan}

\section{OpenEdition}

\section{Journals}

Édition électronique

URL : http://journals.openedition.org/ei/5703

DOI : 10.4000/ei.5703

ISSN : 2553-1891

\section{Éditeur}

Association Économie et Institutions

Référence électronique

Sylvain Vatan, «Faut-il rompre avec les quasi-marchés pour penser la marchandisation ? », Économie et institutions [En ligne], 24 | 2016, mis en ligne le 30 juin 2016, consulté le 21 décembre 2020. URL http://journals.openedition.org/ei/5703 ; DOI : https://doi.org/10.4000/ei.5703

Ce document a été généré automatiquement le 21 décembre 2020.

Revue Économie et institutions 


\title{
Faut-il rompre avec les quasi- marchés pour penser la marchandisation?
}

\author{
Réflexion autour du cas de l'aide à domicile
}

\author{
Sylvain Vatan
}

\section{Introduction}

1 C'est à la fin des années 1980 et au début des années 1990 que s'amorcent les réformes visant à la construction d'un secteur des services à la personne en France. Dans les pays voisins, on assiste à une réforme des services sanitaires, sociaux et médico-sociaux qui affecte également la régulation des services d'aide à domicile. Une littérature se développe alors dans le but d'analyser cette réforme des politiques publiques, qui cherche à instaurer une régulation marchande des activités de service social en général et des activités d'aide à domicile en particulier. Tandis qu'Enjolras (1995) évoque la montée d'un « marché providence » dans l'aide à domicile en lieu et place de l'État, Le Grand (1999, 1991 ; Le Grand et Bartlett, 1993) va reprendre et populariser la notion de quasi-marché à travers l'analyse de la réforme des politiques sociales en Angleterre.

2 L'analyse et la critique du processus de marchandisation dans l'aide à domicile vont alors largement reposer sur la notion de quasi-marché. Pourtant, cette notion souffre d'une imprécision conceptuelle qui rend son utilisation en très grande partie inutile, voire même néfaste lorsqu'elle est mobilisée dans l'objectif d'une critique du processus de marchandisation. En effet, derrière la notion de quasi-marché se tient la notion de marché qui, non seulement n'est pas conceptuellement délimitée, mais dont l'arrimage théorique relève de surcroît de l'analyse économique standard ${ }^{1}$ et se révèle incohérente avec une analyse institutionnaliste du processus de marchandisation. Nous soutenons donc que la notion de marché est un frein à l'analyse institutionnaliste et qu'elle ne permet pas de saisir l'ampleur du processus de marchandisation dans l'aide à domicile. 
3 Nous organisons notre réflexion en quatre temps. Nous revenons d'abord sur les limites de la notion de quasi-marché pour constituer le fondement d'une analyse critique du processus de marchandisation des services sociaux en général et de l'aide à domicile en particulier (1). Nous opérons ensuite un retour aux institutions fondamentales de l'économie monétaire de production capitaliste, en montrant d'une part les difficultés que pose une conception tronquée de la monnaie (2), et en proposant d'autre part de construire une grille d'analyse alternative à partir de la notion de forme marchandise (3). Enfin, nous rendons compte de la pertinence de ce cadre analytique pour appréhender les mutations contemporaines de l'aide à domicile en France (4).

\section{Penser la marchandisation à travers la notion de marché : l'aporie des quasi-marchés}

\subsection{Du constat partagé d'un processus de marchandisation de l'aide à domicile à la recherche d'un cadre conceptuel d'analyse}

La marchandisation est un terme communément employé dans le langage courant pour dénoncer l'évolution de la régulation des services publics. Dans cette utilisation, sa définition a des contours plutôt flous. Elle renvoie à la fois à la privatisation des services publics, à la libéralisation de certains secteurs et la mise en concurrence d'entreprises publiques avec des entreprises privées, ou encore, à la mise en place d'une nouvelle gestion des services publics soumise à l'impératif d'efficacité gestionnaire ; bref, à ce que Bode qualifie de «marchandisation protéiforme» (2007). Pour autant que les frontières de la marchandisation soient floues, ce terme fait directement sens en ce qu'il permet de nommer un certain nombre de pratiques de régulation et d'effets de ces pratiques, qui constituent une réalité de l'évolution contemporaine de l'État social. Si la marchandisation revêt une certaine pertinence au sens commun, son analyse économique nécessite le recours à une définition conceptuelle, qui s'avère en réalité problématique.

Dans le cas de l'aide à domicile, Enjolras annonçait dès le milieu des années 1990 « une marchandisation accrue de ces services et l'avènement d'un véritable "marché providence " " (Enjolras, 1995, p. 7-8). Un certain nombre de recherches, notamment dans le cadre de comparaisons internationales (Laville et Nyssens 2001; Bode 2007; Gardin et al 2010 ; Fraisse et Nassaut 2010 ; Degavre et Nyssens2012), se sont inscrites dans le prolongement des travaux d'Enjolras et évoquent la marchandisation de l'aide à domicile à travers sa mise en marché. Si elles font état d'une tendance transversale à l'adoption de réformes orientées vers le marché, elles insistent néanmoins sur une évolution multiforme à travers les pays, en raison des spécificités des régimes de prise en charge héritées de l'histoire des cadres nationaux de déploiement de l'État social.

Bien qu'elles identifient une marchandisation au sens commun, ces études ne parviennent cependant pas à clarifier sémantiquement et conceptuellement le phénomène qu'elles analysent. En effet elles ne cessent de balancer entre la notion de marchandisation et celle de marchéisation. Dans leur recherche internationale sur l'évolution comparée des régimes de prise en charge de l'aide à destination des personnes âgées, Degavre et Nyssens (2012) utilisent alternativement les termes de commodification et marketization $^{2}$ pour qualifier l'évolution des régulations. Le terme commodification renvoie largement au phénomène de monétisation. Il est employé pour qualifier les effets 
de certaines politiques publiques qui visent à transformer, par le versement de prestations en espèce auprès des familles, le travail d'aide informel en travail rémunéré. Le terme de marketization est quant à lui plutôt employé pour évoquer l'évolution des formes de régulation des activités professionnelles (activités déjà monétisées). Ainsi, la grille d'analyse, mobilisée dans le cadre du projet de comparaison internationale, invite les contributeurs nationaux à étudier le processus de mise en marché des activités d'aide à travers six dimensions (2012, p. 215) :

- l'augmentation de la part du coût supporté par l'usager

- l'augmentation du financement privé de l'aide (non encadré par les politiques publiques)

- l'accroissement de la concurrence entre fournisseurs des services d'aide

- le recours à des procédures contractuelles

- l'accroissement de la part des entreprises lucratives parmi les fournisseurs de services d'aide

- le recours à l'évaluation et la comparaison de la performance des services d'aide

Ce qui est ici nommé mise en marché ou "marchéisation» est repris dans d'autres travaux sous la terminologie de marchandisation. Ainsi, pour Gardin, Nyssens et Minguzzi (Gardin et al, 2010, p. 514), "le secteur du care se caractérise entre un mouvement généralisé vers la marchandisation et une "dépendance de sentier» [...]. En effet, l'analyse de l'impact de l'introduction des quasi-marchés dans différents pays montre que ce mouvement de quasi-marchandisation du care prend une configuration différente suivant la tradition de régime de care». Malgré la pertinence de l'idée qui est ici développée sur les divers horizons de déploiement de la marchandise ${ }^{3}$, ce passage est symptomatique de la superposition entre marché et marchandise. Le quasi-marché se décline en une quasi-marchandisation, alors qu'il est justement censé être le support de la marchandisation (non pas de la quasi-marchandisation). Il ne s'agit pas de pointer ici une simple question de sémantique ou de traduction. En effet, l'usage des mots et le choix des concepts auxquels ils renvoient s'inscrivent au sein de paradigmes et ouvrent des pistes théoriques pour comprendre la réalité à partir de représentations conceptuelles. Or, fonder la marchandisation sur la notion de marché - à travers celle de quasi-marché -, c'est finalement inclure l'analyse de la marchandisation dans le cadre de l'analyse économique standard et de ses extensions. L'analyse théorique des quasi-marchés en fournit l'illustration.

\subsection{Ancrage et limites théoriques des quasi-marchés}

\subsubsection{Conceptualisation des quasi-marchés}

8 A la suite de Williamson (1973) ${ }^{4}$, Le Grand reprend et cherche à conceptualiser la notion de quasi-marché dans le cadre des travaux qu'il poursuit sur l'analyse des réformes des politiques sociales menées en Grande Bretagne (Le Grand 1991, 1999 ; Le Grand et Bartlett, 1993). Les quasi-marchés sont alors définis par leurs divergences vis-à-vis du «marché conventionnel ", à la fois du côté de l'offre et du côté de la demande (1993). Du côté de l'offre, s'il existe bien une concurrence entre différents producteurs ou fournisseurs de services, ces producteurs ne sont néanmoins pas nécessairement des entreprises privées qui cherchent à maximiser leur profit. Du côté de la demande, si l'usager est censé bénéficier du libre choix du fournisseur du service, son pouvoir de consommateur est limité par le fait qu'il n'est pas, en général, le financeur du service (découplage entre consommation et financement du service). 
9 Les quasi-marchés sont ainsi positionnés par rapport à un «marché conventionnel » qui n'est lui-même pas défini, mais dont les caractéristiques apparaissent au fil de la comparaison que font les auteurs entre la réalité des quasi-marchés et la représentation qu'ils se font $\mathrm{du}$ "marché conventionnel ». Il se dégage alors un faisceau d'éléments pour caractériser ce marché conventionnel. Du point de vue des agents, il est composé d'entreprises privées lucratives qui cherchent à maximiser leurs profits dans un environnement concurrentiel, et de consommateurs qui formulent librement leur demande sur la base de préférences individuelles. Du point de vue de la régulation, le " marché conventionnel » permet l'établissement de prix agissant comme des signaux pour une allocation efficace des ressources, en révélant pleinement les préférences des consommateurs et en stimulant les comportements des producteurs.

10 En définitive, le «marché conventionnel » renvoie donc au marché de concurrence pure et parfaite et au modèle de base de la microéconomie néoclassique (Guerrien, 2004). Le cadre de l'analyse économique néoclassique se tenant derrière la référence au "marché conventionnel ", c'est assez logiquement que le recours à la notion de quasi-marchés conduit les auteurs à mobiliser le cadre théorique de l'économie des coûts de transaction. Une fois les critères de performance fixés, les auteurs parviennent à la conclusion que les quasi-marchés sont efficaces :

- s'il y a une structure concurrentielle du côté de l'offre et de la demande ;

- s'il y a une information parfaite et indépendante concernant les coûts pour les fournisseurs et la qualité pour les financeurs ;

- si les coûts de transaction sont minimes - notamment ceux liés à l'incertitude - ;

- si les fournisseurs sont motivés, au moins en partie, par des considérations financières et les financeurs par l'intérêt des usagers ;

- s'il n'y a pas d'incitation à l'écrémage par les fournisseurs des utilisateurs les plus coûteux au profit des utilisateurs les moins coûteux ;

11 En effet, puisque l'efficacité est réalisée par le «marché conventionnel », Legrand et Bartlett en concluent de façon tautologique que le quasi-marché atteint ses objectifs s'il parvient à répliquer les conditions et les hypothèses $d u$ " marché conventionnel $»^{5}$. Même s’ils adoptent une position critique vis-à-vis des quasi-marchés, la référence au "marché conventionnel » conduit logiquement à concevoir les instruments de la théorie des incitations comme des vecteurs d'amélioration de l'efficacité : le succès d'une réforme serait alors lié à la complétude des contrats et au calibrage de la tarification (dont les auteurs montrent néanmoins qu'il est hautement improbable).

12 Alors que nombre de travaux soulignent que les quasi-marchés sont les vecteurs d'une déstabilisation des régulations et des équilibres sociaux (Glennerster et Le Grand, 1995 ; Laville et Nyssens, 2001 ; Fraisse et Nassaut, 2010 ; Gallois, 2012a, 2012b, 2012c), il existe, selon nous, une incohérence à vouloir réaliser une analyse critique du processus de marchandisation à travers une notion de marché non conceptualisée, qui ne peut théoriquement que conclure à la légitimation des pratiques du New Public Management.

13 Nous adhérons donc au jugement de McMaster selon lequel les quasi-marchés relèvent d'une terminologie flottante, dont l'ancrage dans l'économie des coûts de transaction induit un certain nombre de prescriptions de politiques économiques qui se dégagent du paradigme de l'économie de l'information et de la théorie des incitations (McMaster, 2002, p. 770). 


\subsubsection{Incohérence et limites théoriques des quasi-marchés}

14 D'un point de vue théorique, on peut se poser la question de savoir ce qui fonde l'homogénéité des quasi-marchés par rapport aux marchés. Faut-il voir dans ces objets étudiés des marchés imparfaits, au sens de marchés où la concurrence et/ou l'information sont imparfaites? Ou, faut-il réellement comprendre les quasi-marchés comme des presque-marchés, c'est-à-dire comme des espaces qui ne sont pas des marchés ${ }^{6}$ ? Dans les deux cas, la notion de quasi-marché pose problème. D'un côté, si les quasimarchés sont des marchés imparfaits, parce qu'ils s'éloignent du modèle de référence et de ses hypothèses restrictives, on ne voit pas l'intérêt et l'apport de la notion de quasimarché par rapport à la littérature abondante sur la concurrence imparfaite. D'un autre côté, si les quasi-marchés sont des presque-marchés, c'est-à-dire des non-marchés, alors la référence au "marché conventionnel» n'a pas de sens, dans la mesure où son irréalisme conduit nécessairement à conclure qu'aucune forme institutionnelle observable n'est un marché et donc, par extension, que toutes les formes institutionnelles entrent dans cette catégorie du quasi-marché. Comme le souligne Rosenbaum (2000) à propos de la conceptualisation du marché, si la définition d'une forme institutionnelle ne permet pas de discriminer cette forme des autres, alors le concept est vide de sens en plus d'être inopérationnel.

D'un point de vue méthodologique, il existe également un problème de cohérence posé par le recours au "marché conventionnel ». Ainsi, la volonté de comparer les quasi-marchés au «marché conventionnel » relève d'une démarche positive et s'inscrit dans le souci de réalisme de l'économie institutionnaliste. À l'image des travaux mentionnés sur l'aide à domicile, les analyses des quasi-marchés s'inscrivent dans la volonté de rendre compte du réel, à travers une mise en perspective des formes institutionnelles de régulation des activités. L'analyse des régimes de prise en charge de l'aide mobilise ainsi une approche historique, sociologique et politique qui ne trompe pas sur la volonté de ces travaux de rendre compte de la réalité à partir des observations. Mais, cette démarche perd tout son sens dès lors que la réalité est mise en comparaison avec un modèle qui, non seulement n'est pas réaliste, mais ne se conçoit même pas comme un idéal-type (McMaster, 2002, p.770). Il y a donc une incohérence entre l'objectif poursuivi d'une analyse du changement des formes institutionnelles de régulation des échanges et la référence à un " marché conventionnel » anhistorique.

16 Au-delà de ces limites théoriques, il convient d'aborder la question de la pertinence empirique de cet outil.

\subsection{L'aide à domicile à travers les quasi-marchés : quelle pertinence empirique?}

17 Concrètement, les analyses en termes de quasi-marché ont tendance à centrer leur attention sur les éléments de construction de la concurrence. Dans cette perspective, deux éléments sont essentiels : i.la libéralisation des activités d'aide à domicile pour activer la concurrence du côté de l'offre et ii.la solvabilisation de la demande permettant de modifier les préférences des consommateurs et ainsi d'activer le jeu concurrentiel.

Pour ce qui est du champ d'activité soumis à la régulation tutélaire de la puissance publique, l'analyse par les quasi-marchés ne permet d'appréhender le déploiement de la 
marchandisation que par le biais de la contractualisation des autorités publiques avec des prestataires de services en concurrence. Dans le cas d'une régulation tutélaire "classique» (où les services prestataires ne sont pas mis en concurrence pour la contractualisation), les analyses par le quasi-marché sont alors inopérantes pour identifier le processus de marchandisation. Les deux principales voies de la marchandisation sont donc la contractualisation des autorités publiques et la mise en place, sous des formes diverses, d'allocations directes aux usagers pour qu'ils choisissent leur prestataire et exercent ainsi une pression concurrentielle sur les organismes.

"The process of marketisation"

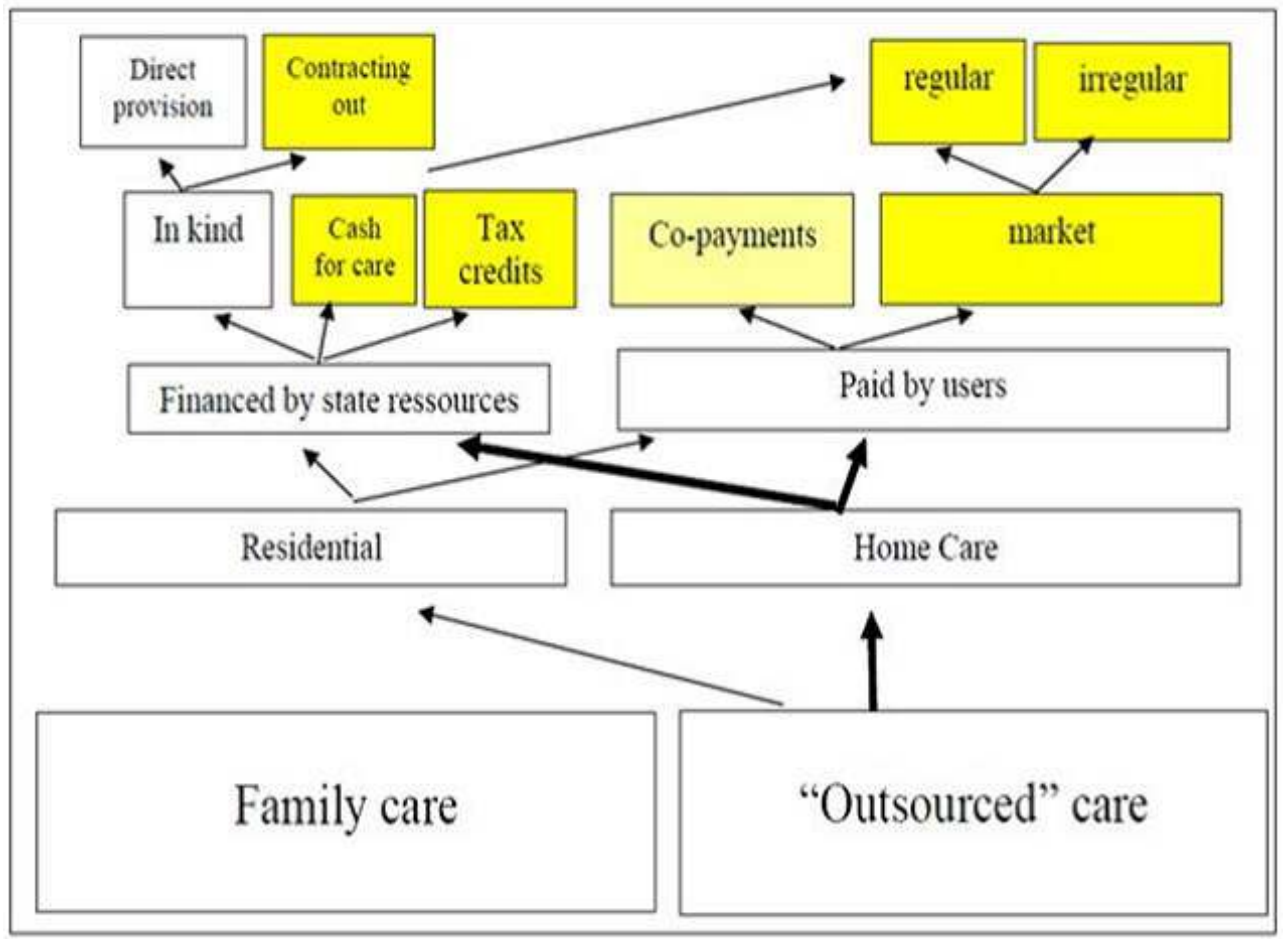

Note : Les cadres colorés constituent, d'après leurs auteurs, les instruments du déploiement de la marchandisation. Source : (Degavre et Nyssens, 2012, p. 105)

Cette identification des ressorts du processus de marchandisation conduit Degavre et Nyssens à considérer, dans leur analyse comparative des systèmes nationaux d'aide à domicile aux personnes âgées, que l'aide à domicile se trouve largement en dehors du processus de marchandisation en Belgique puisque la régulation tutélaire exercée par les financeurs publics i.y empêche la concurrence entre organismes, ii.exclut les organismes à but lucratif de ce champ d'activité, et iii.encadre fortement le travail. Seule l'activité des services domestiques, totalement libéralisée du point de vue de l'entrée des offreurs sur le marché et solvabilisée par la mise en place de titre-services, est concernée par le mouvement de marchandisation lié à la mise en place du quasi-marché des titresservices.

Cette séparation concurrentielle entre les deux champs d'activité (services domestiques et aide à domicile), qui n'existe juridiquement plus en France depuis 2006, conduit alors les analyses par le quasi-marché à une conclusion différente de celle qui prévaut pour le cas Belge. La marchandisation de l'aide à domicile se réaliserait bel et bien en France, et ce par le déploiement de la concurrence du champ associatif et public local (représentant 
les acteurs historiques du champ) par le secteur privé lucratif nouvellement entré dans le champ avec l'affaiblissement des contraintes juridiques. Cette conclusion ne nous paraitt pas rendre véritablement compte de la réalité.

\section{Une activité très segmentée et une concurrence en trompe l'œil}

Le « coup de force politique » du plan Borloo de 2005 visait à la construction d'un grand secteur libéralisé des services à la personne, au sein duquel était insérée l'aide à domicile. Il a contribué à alimenter une littérature importante autour de la mise en concurrence, par les services domestiques, des activités relevant du champ social et médico-social, cette concurrence menaçant la pérennité des acteurs historiques (Devetter et al., 2008, 2009 ; Petrella, 2012; Gallois, 2013) 7 . Du reste, les premières années suivant la mise en place du plan Borloo ont vu l'explosion de la création d'entreprises de services à la personne et de leur activité, puisqu'elles sont passées d'un peu moins de 300 entreprises et de 1,2 millions d'heures d'activité annuelle à près de 18000 entités et 71 millions d'heures (Ould Younes, 2010, 2013).

Mais, cette forte croissance doit être remise dans la perspective du faible poids que ces entreprises représentent dans l'activité en direction des personnes fragiles. Cette fixation des entreprises lucratives, en dehors de ce qui constitue le cœur de l'activité historique de l'aide à domicile, a été relevée dans divers travaux (Jany-Catrice et Vatan, 2011 ; Vatan, 2013; Pinville et Poletti, 2014; Branchu et al, 2015) et peut être étayée à partir des données de la base Nova, référençant les organismes agréés d'aide à domicile. Ainsi, en 2011, ces entreprises ne réalisaient qu'à peine plus d'un tiers de leur activité auprès des publics fragiles, activité qui ne représentait que 13,5\% de l'ensemble des heures prestées auprès de ces publics.

Répartition du nombre et de l'activité des organismes d'aide à domicile selon leur statut en 2011

\begin{tabular}{|l|c|c|c|}
\hline & Associations & CCAS & Entreprises \\
\hline Titulaires de l'agrément de qualité* & 4519 & 1148 & 2402 \\
\hline & $\begin{array}{c}\text { Secteur non } \\
\text { lucratif }\end{array}$ & $\begin{array}{l}\text { Secteur } \\
\text { lucratif }\end{array}$ \\
\hline Volume d'activité (millions d'heures) ** & 163 & 25,4 \\
\hline $\begin{array}{l}\text { Part de l'activité de ces organismes } \\
\text { dédiée aux publics fragiles (hors garde } \\
\text { d'enfants) ** }\end{array}$ & $69 \%$ & $36 \%$ \\
\hline
\end{tabular}

Lecture : En 2011, 4519 organismes de services à la personne agréés qualité relevaient du champ associatif. Le secteur non lucratif composé des associations et des CCAS cumulait 163 millions d'heures d'intervention au domicile de personnes fragiles ce qui représentait $69 \%$ de son activité prestataire.

Source : * Base «nOva » 2011. ** Base «nOva » 2011 ; calculs de l'auteur d'après Ould-Younes (2013)

Bien que les données sur les organismes autorisés (relevant du champ de la régulation tutélaire) soient plus rares, il faut souligner que la plupart de l'activité à destination des personnes fragiles se concentre dans le champ de l'autorisation. Ainsi, en dépit des 
disparités territoriales liées aux différentes politiques départementales de régulation de l'activité, l'étude de la commission d'évaluation de l'assemblée nationale a montré que dans les trois quarts des départements enquêtés, les services autorisés réalisent plus des deux tiers de l'activité menée au titre de l'Allocation personnalisée d'autonomie (Pinville et Poletti, 2014, p. 180). Ces conclusions corroborent les résultats issus d'une enquête réalisée dans la région Nord-Pas-de-Calais, montrant que le champ de l'autorisation regroupait $74 \%$ des emplois des organismes habilités à intervenir auprès des personnes âgées et concentrait $73 \%$ de l'activité en termes d'heures d'intervention (Vatan, 2014, p. 477).

Encadré 1. Autorisation et agrément dans le champ de l'aide à domicile.

En France, l'aide à domicile aux personnes fragiles se caractérise par l'existence de deux régimes distincts de régulation:

L'autorisation est un régime de régulation fondé sur une logique d'action sociale. Elle est délivrée par les Conseils départementaux, en charge de la conduite de l'action sociale. Elle ouvre droit à une tarification administrée, sur la base des règles de la tarification sociale et médico-sociale, qui doit financer les établissements et services au coût de production réel.

Le régime de l'agrément est un régime de régulation fondé sur une logique d'emploi. Il ne déclenche pas la prise en charge des coûts de production réels par la tarification administrée mais ouvre droit à un régime fiscal spécifique, mis en place durant les années 1990 pour soutenir le développement des emplois familiaux.

Depuis la mise en place d'un droit d'option en 2006 (prévu dans le cadre du plan Borloo), l'autorisation du Président du Conseil départemental n'est plus obligatoire pour intervenir auprès des publics fragiles dans le cadre des dispositifs de prise en charge du maintien de l'autonomie. L'agrément préfectoral - beaucoup moins contraignant - suffit désormais pour intervenir dans le cadre des dispositifs de prise en charge. Le cadre juridique a évolué d'un régime cumulatif (agrément + autorisation) vers un régime alternatif. Ce régime, qui a connu beaucoup d'évolutions au cours des vingt dernières années, est encore appelé à se modifier puisque la loi sur le vieillissement en discussion prévoit une nouvelle refonte de ce système.

Depuis la mise en place d'un droit d'option en 2006 (prévu dans le cadre du plan Borloo), l'autorisation $\mathrm{du}$ Président du Conseil départemental n'est plus obligatoire pour intervenir auprès des publics fragiles dans le cadre des dispositifs de prise en charge du maintien de l'autonomie. L'agrément préfectoral - beaucoup moins contraignant - suffit désormais pour intervenir dans le cadre des dispositifs de prise en charge. Le cadre juridique a évolué d'un régime cumulatif (agrément + autorisation) vers un régime alternatif. Ce régime, qui a connu beaucoup d'évolutions au cours des vingt dernières années, est encore appelé à se modifier puisque la loi sur le vieillissement en discussion prévoit une nouvelle refonte de ce système.

La concurrence dans l'aide à domicile est donc un phénomène tout relatif (et par ailleurs très territorialisé). Il est en outre très complexe dans la mesure où les liens entre intensité concurrentielle, sensibilité des prix et variation de l'activité ne s'inscrivent pas dans les conclusions du modèle du "marché conventionnel». Bien que peu d'études renseignent sur la question, l'intensité concurrentielle semble avoir très peu d'effet sur 
les prix tandis que le niveau d'activité est quant à lui très sensible aux prix (Vatan, 2013). Les usagers des services d'aide à domicile ne semblent pas prompts à changer de service prestataire pour des questions de prix et, lorsque les tarifs s'élèvent et que les restes à charge ${ }^{8}$ sont plus importants, ils ont plutôt tendance à accentuer le recours à l'aide informelle (celle de leurs proches) ou à passer par quelques heures en emploi direct (Gucher et al., 2011)'.

Si la concurrence a imprégné les discours et les modes de penser du champ, notamment dans l'adoption et le renforcement de démarches commerciales (Fraisse et Gounouf, 2008; Jany-Catrice, 2010; Petrella et Richez-Battesti, 2010; Vatan, 2013), on peut néanmoins soutenir qu'elle n'a touché qu'à la marge l'activité historique d'aide à domicile. Le secteur des services à la personne ne s'est pas construit sur une interpénétration des activités mais bien plutôt sur une construction-segmentation des activités (et corrélativement des publics). Malgré les incitations, voire les injonctions institutionnelles à la diversification, le secteur des services à la personne reste donc marquée par une «dualité sectorielle étanche » que nous considérons au regard des volumes d'activité, à la différence de Gallois et Nieddu, comme une réalité et non comme une "hypothèse » (Gallois et Nieddu, 2015, para. 27).

Pour Gallois (2012a), s'il existe bien différents types de modèles productifs liés à la trajectoire historique des organismes, leur reconfiguration est largement liée à l'insertion des organismes dans le quasi-marché des services à la personne, à leur plongée dans un nouvel environnement concurrentiel qui les a contraint à modifier leur stratégie de compétitivité. Pourtant, comme elle le fait remarquer, "les acteurs historiques, qui sont tarifés, ont cherché à diversifier leurs sources de financements en développant de nouveaux services, élargissant leur gamme à l'ensemble des activités de services à la personne [...], mais sans que ces activités ne soient significatives de leur activité (tout au plus 10 à $15 \%$ )» (op. cit., p. 256).

\section{Une contractualisation éloignée du modèle concurrentiel}

Dans l'analyse par les quasi-marchés, c'est à travers le phénomène de contractualisation que la marchandisation est appréhendée lorsque la collectivité publique jouait historiquement un rôle déterminant dans la délivrance de l'aide à domicile. L'externalisation de la prise en charge et la mise en concurrence des opérateurs avec lesquelles la puissance publique contracte deviennent alors les leviers de la marchandisation. Cette mise en concurrence peut être soutenue ou non par la mise en place d'une solvabilisation directe des usagers et l'exercice du libre choix. Dans ce cas, la pression concurrentielle se fait non seulement par l'intermédiaire d'une sorte de «marché public » de l'action sociale, mais également par l'intermédiaire des usagers qui arbitrent entre les différents organismes délivrant le même type de services.

En France, si la contractualisation entre les organismes prestataires et les tutelles financières ${ }^{10}$ se développe, elle est encore loin de reposer sur un modèle de mise en concurrence généralisée. Depuis la loi de 2002 de rénovation sociale et médico-sociale, les Conseils départementaux peuvent contractualiser avec les organismes sociaux et médicosociaux dans le cadre de Contrats Pluriannuels d'objectifs et de Moyens (CPOM). Dans les faits, ces contrats n'ont commencé à être signés qu'à la fin des années 2000. Surtout, la mise en pratique de la contractualisation ne s'intégrait pas dans une logique de mise en concurrence mais bien plutôt de renforcement du contrôle financier exercé par la tutelle sur l'organisme, dans un contexte de difficultés financières pour les organismes d'aide à 
domicile et d'explosion de la charge financière des Départements liée à l'action sociale. Ce n'est qu'en 2009 avec la loi HPST ${ }^{11}$ que la logique concurrentielle a été intégrée au cadre existant de la contractualisation. En effet, ce nouveau cadre juridique prévoit désormais que l'autorisation délivrée par les Conseils départementaux i.se réalise obligatoirement par la signature d'un CPOM et ii.procède d'un appel à projet lancé conjointement par l'Agence régionale de santé et les Conseils départementaux. Les premières autorisations ayant été délivrées au milieu des années 2000 (pour une durée de 15 ans), la situation actuelle des organismes autorisés est loin de correspondre au modèle de contractualisation dans le cadre de marchés publics. S'il existe une forme de concurrence entre les organismes autorisés, celle-ci n'est pas liée à la contractualisation avec les tutelles et, de l'aveu même des responsables d'organismes, elle a toujours existé dans le champ.

La transformation profonde vécue par le champ de l'aide à domicile, très largement composé des services autorisés, ne parvient donc pas à être expliquée par les analyses en termes de quasi-marché. De notre point de vue, le recours à une analyse par le marché (ou le quasi-marché), au fondement de laquelle se retrouve la question du degré de perfection des marchés (et donc de " pureté » de la concurrence), conduit à ne penser la marchandisation qu'à travers le prisme concurrentiel et celui de la construction institutionnelle du marché (le plus concurrentiel possible). Or, la reconfiguration des modèles productifs, ainsi que la reconfiguration de la forme du rapport salarial à l'œuvre dans l'aide à domicile procèdent d'un mouvement qui se construit largement en dehors du modèle de concurrence et qui relève pourtant bien selon nous d'un processus de marchandisation.

\section{La monnaie : condition nécessaire mais non suffisante de la relation marchande}

31 La question de la monnaie est intimement liée à celle de la marchandisation et de la relation marchande. Que la monnaie soit liée à la marchandise et appréhendée dans le cadre des rapports sociaux de production capitalistes chez Marx, ou qu'elle déborde le mode de production capitaliste et soit posée comme le support de tout rapport marchand en dehors de la spécificité des rapports sociaux de production (Orléan et Aglietta, 2002; Orléan, 2008), l'extension de la marchandise ou de la logique marchande impose une réflexion autour de la question monétaire. L'analyse de l'extension marchande dans le champ de l'aide à domicile et, plus largement, dans l'ensemble des services sociaux ne déroge pas à cette règle. Que ce soit dans le cadre de l'économie plurielle ou dans les travaux relevant plus de l'économie du genre (s'intéressant particulièrement à la question des travaux domestiques et d'aide à domicile), l'analyse de la marchandisation entretient des liens étroits avec la conception de la monnaie. Bien que ces deux courants (dialoguant entre eux) campent sur deux visions polaires de la monnaie, nous pensons qu'ils fondent leur position sur une même conception réductrice, superposant monnaie et marchandise. 


\subsection{Des deux visions polaires de la monnaie dans les analyses du travail d'aide}

\subsubsection{L'économie plurielle : la monétisation comme atrophie du lien social}

En France, le cadre de l'économie plurielle a principalement été développé par Laville (Eme et Laville, 1999 ; Laville, 2000, 2003, 2008). Il s'intéresse particulièrement au tiers secteur et au rôle des associations dans la gestion des services de proximité et des activités à fort contenu relationnel et politique. Les comparaisons internationales sur l'évolution des services d'aide à domicile s'inscrivent dans cette perspective (Laville et Nyssens, 2001; Degavre et Nyssens, 2012) et mettent l'accent sur le welfare mix, c'est-àdire sur l'articulation entre les différentes sphères de production et les différentes modalités de délivrance des activités d'aide.

En partant du constat que ces activités sont dispensées à travers divers espaces sociaux de régulation des échanges (marché, famille, État), régis selon différentes formes de rationalité, ce courant de pensée insiste sur le rôle du tiers secteur (les organismes privés à but non lucratif) dans l'articulation de ces formes de rationalité et sa capacité à porter une économie plurielle, mêlant les différentes formes sociales d'allocation des ressources économiques (l'échange, la redistribution, la réciprocité et l'administration domestique). L'hybridation de ces formes d'allocation est réputée désirable, en ce qu'elle permet d'équilibrer les logiques caractérisant les divers lieux de production et d'échange: poursuite de l'intérêt individuel pour l'économie marchande (marché), poursuite de l'intérêt général pour l'économie non marchande (État) et construction du lien social pour l'économie non monétaire (administration domestique et réciprocité) (Laville, 2008, pp. 48-49).

Dans cette perspective, "l'économie non monétaire correspond à l'économie dans laquelle prime le souci de l'autre, exprimé à travers une complémentarité et une interdépendance attestées par des prestations non monétaires et renvoyant à un lien social, soit volontaire dans le cas de la réciprocité, soit hérité dans le cas de l'administration domestique» (Laville, 2008, pp. 48-49). Le caractère non monétaire attesterait donc en quelque sorte du "véritable " lien social ou, plutôt, du lien social le plus fort, celui fondé sur la réciprocité et le don ${ }^{12}$. Cette vision suggère que la dimension relationnelle se diluerait dans la monnaie, bien que dans la sphère publique et les activités menées sous la tutelle de l'État social, « les valeurs démocratiques et les valeurs de solidarité peuvent jouer un rôle bien plus important " que dans le marché (Laville, 2008, pp. 48-49).

35 Cette conception nous semble réductrice en ce qu'elle considère la monnaie dans sa fonction d'intermédiaire des échanges et passe à côté du fait monétaire en tant que fait social total. Or, la monnaie dépasse de loin sa fonction d'échange. Elle est un lien entre les hommes, elle symbolise la dette qui les lie à la totalité sociale et qui circule entre eux (Aglietta et Orléan, 1998). L'activité monétaire ne porte donc pas en soi la marque d'un lien social atrophié, bien au contraire. La monnaie est non seulement un marqueur du lien social, mais elle est véritablement constitutive de ce lien. Dans le cas d'une économie monétaire de production, la monnaie est le sceau du travail social. Elle a le double statut $i$. d'intégrateur social et politique du travail dans la communauté politique élargie (le travail est reconnu socialement hors du champ familial ou communautaire) et ii.de 
libérateur social et économique, puisqu'en vertu de sa liquidité parfaite, elle permet de solder ses dettes.

La première dimension de la monnaie - intégrateur social et politique - est oubliée par les tenants de l'économie plurielle, qui perçoivent plus la monnaie comme un vecteur de désintégration que de reconnaissance sociale ${ }^{13}$. Si cette première dimension est oubliée, c'est parce que la seconde - celle de libérateur social et économique - est hypertrophiée et que le caractère libérateur de la monnaie fait l'objet d'un réductionnisme aux caractéristiques de l'échange marchand. En effet, bien que l'économie plurielle distingue la logique inhérente à la sphère marchande de celle de la sphère non marchande, elle les oppose toutes les deux à la logique du souci de l'autre, du lien solidaire, qui serait propre à la sphère non monétaire. Le caractère libérateur de la monnaie affaiblirait le lien social entre les hommes. Certes, la monnaie, par son caractère libérateur, est le support nécessaire de la relation marchande, dans laquelle l'individu qui paye est quitte, croit pouvoir se débarrasser de toute autre dimension relationnelle et s'extirper du rapport social qui enserre cette relation d'échange. Mais, le caractère libérateur de la monnaie ne peut être réduit à l'image de l'échange marchand, car s'il est émancipateur des tutelles communautaires et des contraintes sociales du modèle réciprocitaire, il ne rompt pas les liens sociaux mais les transcende dans l'espace politique. En ce sens, les travaux de Blanc (2007) sur les monnaies sociales locales illustrent bien la dimension politique de la monnaie et le rôle que jouent certaines propriétés de la monnaie dans l'éloignement des échanges de la logique marchande, dépassant la simple dimension de « liberté frappée ».

\subsubsection{La nouvelle sociologie économique américaine et l'économie du genre : vertus de la monnaie ou de la marchandisation?}

Dans une approche qui cherche à insister davantage sur la question du genre que sur celle du tiers secteur, les travaux de Folbre (England et Folbre, 1999; Folbre et Nelson, 2000 ; England et Folbre, 2005) ou de Zelizer $(1994,2008,2011)$ conduisent également à inscrire les activités de travail familial et/ou domestique dans la perspective d'une économie mixte où s'enchevêtrent les rationalités et les logiques d'action. Pour ces auteures, l'introduction de la monnaie dans les rapports humains ne provoque pas nécessairement un effritement $d u$ sens du travail et du lien social dans les activités concernées (notamment dans les activités d'aide). Pour Zelizer, le marquage de l'argent et l'affectation spécifique de la monnaie au sein des foyers traduisent une signification sociale de l'argent, qui empêche de voir la monnaie comme un instrument neutre et destructeur du lien social (Zelizer, 1994, p. 142). Pour Folbre et Nelson (2000), non seulement la monnaie ne détruit pas le lien social ni ne pervertit les activités qui sont monétisées, mais elle présente un certain nombre d'avantages. Concernant le travail d'aide (familial et/ou domestique), son externalisation et sa réalisation au sein des activités monétaires lui permettraient d'être réalisé dans de meilleures conditions : i.en libérant les femmes du fardeau que peut constituer ce travail d'aide dans la sphère familiale, ii.en spécialisant et en professionnalisant des activités qui seraient désormais réalisées par des personnes plus qualifiées et iii.en rendant visible un travail des femmes, qui était jusqu'à présent caché et naturalisé au sein de la cellule familiale.

Si ces travaux ont le mérite de ne pas donner une vision négative de la monnaie, celle-ci est cependant superposée à la notion de marché et à l'économie marchande. Dès lors, puisque ces auteures soulignent que la monétisation du travail d'aide n'est pas néfaste et qu'elle peut même être positive, elles en concluent que sa mise en marché ne doit pas être 
combattue. En réalité, la monétisation est réduite à la marchandisation/marchéisation des activités ${ }^{14}$, si bien que c'est la mise en marché des activités d'aide et de soin qui est censée produire les avantages escomptés de sa transformation en activité monétaire ${ }^{15}$.

S'appuyant sur l'analyse des réseaux sociaux et une approche culturaliste (Convert et Heilbron, 2005 ; England et Folbre, 2005), cette nouvelle sociologie économique défend l'idée que l'encastrement culturel et structural des marchés éloigne l'échange marchand de la rationalité froide du calcul utilitariste, et autorise une re-personnalisation du lien marchand. Cette vision, fondée sur une « conception « intimiste» du lien social » (Chantelat, 2002), empêche ces analyses de saisir la dimension sociale de l'économie marchande moderne qui se trouve dans ses institutions fondamentales (Orléan, 2003).

\subsection{La monnaie comme vecteur de socialisation du travail}

Marchande ou non, la production devient monétaire du fait même qu'elle s'inscrit dans le cadre d'un travail social. Dans une économie monétaire de production, caractérisée par la division sociale du travail, la socialisation du travail se fait par l'intermédiaire de la monnaie qui sanctionne son utilité sociale : le rôle social des travailleurs dans le mode de production est reconnu par l'institution monétaire. À l'inverse, dans l'économie privée (familiale ou communautaire), le caractère social du travail se manifeste directement dans l'organisation de la communauté et de la production. Cette fonction socialisatrice de la monnaie, vis-à-vis du travail, constitue l'un des apports de la critique de l'économie politique faite par Marx, ce dernier insistant sur le fait que le «travail de l'individu isolé [...] ne devient social qu'en prenant la forme de son contraire immédiat, la forme de la généralité abstraite » (Marx, 1859, p. 285). Découplée de la théorie de la valeur travail, l'idée n'en demeure pas moins chez Orléan pour qui «dans l'économie marchande, l'existence sociale de toute chose passe par son expression monétaire » (Orléan, 2008).

En permettant de distinguer le travail professionnel du travail domestique, l'introduction de la monnaie offre un cadre analytique pour penser les activités d'aide à domicile. Ainsi, le travail des intervenantes au domicile peut être directement social lorsqu'il est exercé au sein de la communauté familiale, du voisinage, de la communauté religieuse ou de la communauté de travailleurs ${ }^{16}$. L'activité d'aide est alors directement liée à la place qu'occupe chaque individu (aidant et aidé) et au rôle social qui lui est assigné dans la communauté. Dans la société où prévaut la division sociale du travail, c'est-à-dire une séparation entre l'activité productive singulière des individus et leur place dans le corps social, l'utilité sociale des travaux individuels n'est pas inhérente à la structure sociale, mais elle est validée par la monnaie qui agit comme une médiation institutionnelle. Les aides à domicile, auxiliaires de vie ou travailleuses sociales (et l'ensemble des aidantes professionnelles), voient leur travail reconnu socialement et politiquement d'abord à travers la monnaie, qui le pose en tant qu'équivalent général de tous les autres et lui attribue donc l'aspect social.

Le souci de l'autre, la complémentarité et l'interdépendance ne sont pas solubles dans la monnaie. Bien au contraire, la monnaie institue une activité productive en tant que travail social. Elle n'efface pas l'aspect social et interpersonnel de l'activité productive, mais lui attribue une véritable dimension politique. Le caractère libératoire de la monnaie sur le plan économique - sur lequel s'appuie la relation marchande -, également synonyme de libération sociale, coexiste parfaitement avec le rôle intégrateur du travail par la monnaie dans le cadre d'une économie monétaire de production. 
Avant la marchandise et la marchandisation se pose donc le processus de reconnaissance sociale de l'activité productive par la monnaie. S'il ne peut y avoir de marchandise et de rapport marchand sans monnaie, toute activité monétisée n'est pas pour autant « marchandisée ». La monnaie peut exister sans la marchandise, mais la réciproque n'est pas vraie. En somme, la monnaie n'emporte pas la logique marchande, tout comme la logique marchande n'épuise pas le fait monétaire. L'effritement du lien social n'est pas tant lié au fait monétaire qu'à la relation marchande, qu'il nous faut désormais analyser à travers le concept de marchandise.

\section{Refonder la marchandisation sur le concept de marchandise}

Nous ne revenons pas ici sur l'histoire de la marchandise en tant que question fondamentale de l'économie politique et son lien avec la théorie de la valeur (Lapidus, 1986). Nous centrons notre analyse sur la notion de forme-marchandise que nous tirons des travaux de De Vroey (1984) qui, prolongeant les interrogations de Marx et Polanyi, nous semble offrir un cadre d'analyse particulièrement pertinent pour rendre compte des différentes dimensions de la marchandisation.

\subsection{De la marchandise au sens strict à la forme marchandise}

En constatant que si toutes les marchandises (les valeurs) ont une forme prix mais que certaines formes prix ${ }^{17}$ ne sont pas des valeurs (puisqu'elles s'appliquent à des produits qui ne sont pas le fruit du travail), Marx laisse en suspens une question théorique qui sera reprise par Polanyi (1944), s'interrogeant à son tour le statut de ces «marchandises fictives ». Comment certains biens - tels que la terre, la monnaie, la force de travail peuvent devenir marchandises, alors qu'ils ne sont pas le fruit d'un travail ?

À cette question théorique, De Vroey (1984) tranche de façon radicale. Pour lui, si l'on s'en tient à une définition stricte de la marchandise, telle que Marx l'a lui-même échafaudée, ces biens échangés ne sont pas des marchandises. Il propose ainsi de clarifier le concept de marchandise à partir d'une double définition : l'une au sens large, l'autre au sens étroit. Sont marchandises au sens étroit les "valeurs d'usage présentées sur un marché, au moment effectif de leur vente, pour autant qu'elles résultent d'une activité de travail professionnel» (1984, p. 111). Au sens large en revanche, « est [...] marchandise tout ce qui fait l'objet d'une transaction sur un marché et qui, de ce fait, a un prix. Les deux notions, marché et marchandise, se recoupent pleinement. » (1984, p. 110).

De Vroey livre en réalité une distinction entre marchandise et forme marchandise. Ce qui confère la substance de marchandise est la nature du travail (professionnel privé), ainsi que la forme et le moment de la validation sociale (individuelle et ex post via le marché). En revanche, ce qui confère la forme marchandise est l'existence d'un prix comme principal instrument de régulation des échanges. En résumé, «si toute marchandise a un prix, tout ce qui a un prix n'est pas pour autant une marchandise » (1984, p. 111).

48 La polysémie du terme est donc relative, puisqu'il est possible de circonscrire ce qui relève de la marchandise de ce qui n'en est qu'un simple ersatz. Pour clarifier le propos, on nommera marchandises les biens relevant de la première définition et formes- 
marchandises ceux relevant de la seconde définition. Les formes-marchandises font l'objet d'échanges régulés par les prix, mais ne sont pas des marchandises au sens strict ${ }^{18}$.

Dans sa réflexion, De Vroey s'attache principalement à circonscrire la marchandise au sens étroit, afin de requalifier la force de travail comme une non-marchandise. La définition de la marchandise au sens large, après avoir été énoncée, n'est pas véritablement développée. Or, cette différenciation entre la substance marchandise et la forme marchandise nous apparaît pertinente pour appuyer théoriquement le processus de marchandisation et sortir des analyses en termes de quasi-marchés. Nous choisissons donc recourir au concept de forme-marchandise afin de comprendre quelles sont les logiques à l'œuvre dans un champ comme celui de l'aide à domicile. S'il n'existe pas de marchandise travail ou de marchandise terre, il n'en demeure pas moins qu'il existe bel et bien un marché du travail ou un marché de la terre : un espace où les rapports sociaux d'échange sont régulés par des prix. Il existe aujourd'hui un marché de l'aide à domicile, même si ces activités ne peuvent pas encore, pour une large partie d'entre elles, être qualifiées de marchandises au sens strict.

\subsection{La relation marchande et le rôle paramétrique du prix}

\subsubsection{Forme marchandise et relation marchande}

Dans la pensée Aristotélicienne et Marxienne, le concept de marchandise recèle un caractère éminemment social. La marchandise n'est pas un objet mais un rapport social, " son objectivité est toujours un rapport au groupe, construit socialement » (Orléan, 2003, p. 185)

19. Cette conception de la marchandise en tant que rapport social a deux implications majeures. D'une part, leur forme physique n'exclut pas a priori certaines productions du champ de la marchandise : biens ou services peuvent être des marchandises. C'est bien la spécificité des rapports sociaux, contenus dans les activités productives et les productions échangées, qui peut les exclure du champ de la marchandise (la force de travail n'est pas produite pour être échangée, la terre et la monnaie ne sont pas le produit d'un travail professionnel). D'autre part, l'analyse du processus de marchandisation nécessite de caractériser la transformation des rapports sociaux contenus dans les activités de production et d'échange. Analyser la mise en marchandise, c'est mettre en évidence le changement de la logique qui préside à la régulation des activités de production et d'échange. Pour le champ qui nous occupe - l'aide à domicile -, cela signifie que cette activité n'est pas forcément en dehors du champ de la marchandise en raison de son caractère immatériel ou de son contenu relationnel et contextuel. Par la transformation des rapports sociaux qu'elle renferme, cette activité peut tout à fait être marchandisée.

51 La marchandisation est donc un processus de transformation sociale, consistant « à rendre marchande une relation qui ne l'était pas auparavant ", (Guerrien, 2003, p. 121). La relation marchande est généralement présentée à travers les caractéristiques de la forme marchandise, c'est-à-dire de la place centrale du prix dans le processus décisionnel ou la régulation des transactions par les prix.

Marx insiste sur ce point lorsqu'il souligne que «ce qui intéresse tout d'abord pratiquement les échangistes, c'est de savoir combien ils obtiendront en échange de leurs produits, c'est-à-dire la proportion dans laquelle les produits s'échangent entre eux. » (1867, p. 608). La logique marchande consiste donc bien à réduire le contenu (social) de l'échange à un simple rapport (au sens arithmétique) d'échange. La dimension qualitative 
de la relation d'échange s'efface (sans jamais disparaître totalement) derrière un rapport quantitatif, dont seule l'expression monétaire apparaît aux yeux des individus. Les rapports entre les choses - et la forme qu'ils revêtent, c'est-à-dire des prix monétaires ${ }^{20}$ dissimulent les rapports entre les hommes. Le processus de marchandisation repose sur cette logique de réduction des relations d'échange à des rapports quantitatifs entre les choses.

Ce changement de logique, caractéristique de l'échange marchand, est souligné par l'anthropologue Alain Testart (Testart, 2001, p. 726) qui, pour différencier le don de l'échange, en revient à une différenciation entre l'échange marchand et l'échange non marchand.

«Est échange marchand tout échange de marchandises, ou encore tout échange dans lequel les échangistes n'ont pas besoin d'entretenir entre eux d'autre rapport que celui de l'échange, c'est-à-dire encore un échange qui n'est pas intrinsèquement lié ni conditionné par un autre rapport entre les protagonistes. » (Testart, 2001, p. 726).

54 Ce que soulève ici l'anthropologue, c'est l'existence d'un basculement dans la rationalité des échangistes. C'est l'idée même que soutient Orléan (Orléan, 2003, pp. 184-185) pour qui le modèle marchand pur, tel qu'il peut être représenté par le modèle walrassien, repose sur le fait qu'une rationalité paramétrique, fondée sur les prix, peut se substituer à une rationalité stratégique, fondée sur la prise en compte du comportement des autres agents.

«Tout ce que les agents ont à savoir sur la manière dont les autres agissent est intégralement contenu dans les prix. On peut donc dire que, dans le modèle walrassien, les prix constituent une médiation parfaite au sens où ils font parfaitement écran entre les hommes. Ils absorbent toute la substance sociale : le seul rapport aux prix suffit à déterminer complètement la position de chacun des acteurs économiques sans qu'il soit nécessaire pour eux d'entrer en relation directe avec les autres agents, ou même de s'y intéresser. » (Orléan, 2003, pp. 184-185).

Dans ce changement de rationalité réside le principe de séparation marchande qui repose sur la médiatisation de toutes les relations humaines à travers les institutions marchandes (monnaie et marchandise). L'introduction d'un rapport objectivé entre les choses, d'un prix, permet le déploiement d'une rationalité paramétrique caractéristique de la logique marchande. L'intensification du rôle paramétrique ${ }^{21}$ du prix dans la relation d'échange permet de juger de l'évolution du processus de marchandisation.

\subsubsection{Le rôle paramétrique du prix}

Le processus de marchandisation, à partir de la notion de forme marchandise, peut donc être défini comme un processus de renforcement du rôle paramétrique du prix dans les relations d'échange. Nous posons l'idée que ce processus de renforcement doit s'analyser à la fois du point de vue micro et meso-social, mais également d'un point de vue macroéconomique. Si le renforcement du rôle paramétrique du prix est une caractéristique du lien marchand dans le rapport entre échangistes, il est également une caractéristique du fonctionnement macroéconomique de l'économie marchande.

57 En reprenant les trois temps du fonctionnement d'une économie monétaire de production, que sont i.l'anticipation, ii.le financement et iii.la validation de la production (Van de Velde, 2005 ; Poulon, 2008), Harribey (2009) montre que secteur marchand et secteur non-marchand fonctionnent selon ce même circuit. La différence entre les deux 
réside dans le fait que pour le secteur non marchand, l'anticipation se confond avec la validation puisque la décision pour l'État d'engager une production se traduit nécessairement par une validation concomitante dans la mesure où cette validation de la production, ce paiement, est réalisée de façon collective et centralisée. Là où la validation individuelle et décentralisée de la production (validation marchande) est entièrement médiatisée par les prix, qui sont le point gravitationnel de décision des agents, la validation collective et centralisée (validation monétaire non marchande) se fait par l'impôt sur la base d'un choix collectif. L'impôt en tant que "prix socialisé» (Harribey, 2008, p. 129) n'occupe pas de rôle déterminant dans le processus de validation de la production ${ }^{22}$.

En repartant des deux institutions centrales de l'économie monétaire de production que sont la monnaie et la marchandise, on peut ainsi dégager une typologie des activités productives mettant en avant le rôle paramétrique du prix. Ce dernier se révèle alors comme un objet d'analyse pour comprendre le déploiement de la marchandisation.

Typologie des activités productives

\begin{tabular}{|c|c|c|c|}
\hline & \multicolumn{2}{|c|}{ Sphère monétaire } & \multirow{2}{*}{$\begin{array}{c}\text { Sphère } \\
\text { non } \\
\text { monétaire }\end{array}$} \\
\hline & marchande & $\begin{array}{c}\text { non } \\
\text { marchande }\end{array}$ & \\
\hline $\begin{array}{l}\text { Intensité sociale } \\
\text { de l'échange }\end{array}$ & $\begin{array}{l}\text { Faible (voire } \\
\text { nulle dans une } \\
\text { représentation } \\
\text { idéale typique }\end{array}$ & $\begin{array}{l}\text { Elevée (voire } \\
\text { totale dans } \\
\text { une } \\
\text { représentation } \\
\text { idéale- } \\
\text { typique) }\end{array}$ & Totale \\
\hline $\begin{array}{l}\text { Formes } \\
\text { institutionnelles } \\
\text { du taux } \\
\text { d'échange }\end{array}$ & Prix monétaire & Prix monétaire & $\begin{array}{l}\text { Gratification } \\
\text { symbolique } \\
\text { - Echange } \\
\text { par le don }\end{array}$ \\
\hline $\begin{array}{l}\text { Moment de la } \\
\text { validation } \\
\text { sociale de la } \\
\text { production }\end{array}$ & Ex-post & Ex ante & Ex ante \\
\hline $\begin{array}{l}\text { Nature/Forme } \\
\text { de la décision } \\
\text { de validation de } \\
\text { l'activité sociale }\end{array}$ & $\begin{array}{l}\text { Individuelle/ } \\
\text { décentralisée }\end{array}$ & $\begin{array}{l}\text { Collective/ } \\
\text { centralisée }\end{array}$ & $\begin{array}{l}\text { Collective/ } \\
\text { centralisée }\end{array}$ \\
\hline $\begin{array}{l}\text { Type de } \\
\text { rationalité }\end{array}$ & Paramétrique & Stratégique & Stratégique \\
\hline
\end{tabular}




\begin{tabular}{|l|c|c|c|}
\hline $\begin{array}{l}\text { Rôle } \\
\text { paramétrique } \\
\text { du prix }\end{array}$ & $\begin{array}{c}\text { Elevé (voire } \\
\text { total dans une } \\
\text { représentation } \\
\text { idéale- } \\
\text { typique) }\end{array}$ & $\begin{array}{c}\text { Faible (voir nul } \\
\text { dans une } \\
\text { représentation } \\
\text { idéale- } \\
\text { typique) }\end{array}$
\end{tabular} \mid

Source : Auteur.

\section{Une lecture du déploiement de la marchandisation dans l'aide à domicile à travers l'analyse des formes tarifaires}

59 En examinant le rôle paramétrique du prix dans les relations d'échange et la place qu'il occupe dans le processus de validation de l'activité, il est possible de rendre compte des différents espaces de déploiement de la marchandisation dans l'aide à domicile. Le déploiement de la marchandise au sens stricte nous apparaît alors comme limité au regard de ce que nous considérons comme l'échec du plan Borloo. En revanche, l'expansion de la forme marchandise est le véritable vecteur de la marchandisation de l'aide à domicile par la transformation de la tarification administrée.

\subsection{L'échec du plan Borloo ou l'expansion limitée de la marchandise au sens stricte}

En prévoyant un droit d'option entre l'agrément préfectoral et l'autorisation du Conseil général, le plan Borloo visait à ouvrir le champ de l'aide à domicile (relevant de l'action sociale et médico-sociale) au secteur privé lucratif. Il offrait alors la possibilité d'un déploiement du prix marchand dans l'aide à domicile.

61 Nous qualifions ici de prix marchand le prix « librement » fixé par les producteurs et qui constitue la variable déterminante de la validation sociale du travail professionnel. Il se distingue du prix de marché, dans l'analyse économique néoclassique, en ce qu'il est administré par les producteurs et non fixé par la rencontre de l'offre et de la demande. Dans le champ de l'aide à domicile, ces prix concernent les organismes qui bénéficient de l'agrément préfectoral, mais ne sont pas titulaires de l'autorisation délivrée par le président du Conseil général. Ils sont réglementairement encadrés, puisqu'un arrêté ministériel annuel fixe le taux maximal d'évolution des prix. Mais, dans la pratique, ils sont établis sans réelle contrainte pour les producteurs ${ }^{23}$.

Ce sont les producteurs du service qui anticipent l'activité et les usagers qui valident ou non ces anticipations sur la base des prix administrés par les producteurs. En décentralisant et en individualisant la validation sociale de la production, la collectivité s'efface donc de la relation d'échange et, avec elle, une part importante de la dimension politique de l'activité d'aide à domicile qui s'est historiquement construite en tant que relation de service social (Gardin, 2008 ; Noguès, 2008).

Les activités d'aide réalisées dans le cadre de l'agrément et délivrées en contrepartie de prix librement fixés sont donc celles qui renvoient le plus à l'idéal-type de l'activité marchande. Les prix y cristallisent un rapport d'échange désincarné où les relations 
tendent à se réduire à un taux d'échange. La logique collective d'anticipation et de validation d'une production, répondant à des besoins définis politiquement, s'efface derrière une logique individualisée de consommation de service et de validation du travail social décentralisée. Si le financement reste en partie collectif (exonérations fiscales, allocations directes utilisables auprès d'organismes non tarifés), le paiement est privatisé et la réallocation des ressources se fait exclusivement sur la base de prix. L'individualisation et la rationalisation des choix du côté du consommateur (allocation directe couplée à la politique du libre choix), comme du producteur (mise en concurrence entre prestataires), conduisent de plus en plus à dépouiller l'échange de son contenu social et relationnel, pour lui conférer la forme d'un échange de service sur la base de prix. Les activités régies par ce mode de coordination (prix librement administrés) relèvent donc de la sphère marchande et les services constituent des marchandises, même si les rapports sociaux ne sont pas exclusivement réductibles à des rapports quantitatifs.

Il faut noter que ce pan de l'activité - celui de l'agrément - est celui où se concentrent les organismes à caractère lucratif. En effet, si légalement les entreprises à but lucratif peuvent être autorisées et ainsi bénéficier de la tarification administrée, dans les faits, toutes les entreprises se concentrent dans le champ de l'agrément (Vatan, 2013; Pinville et Poletti, 2014). Certes, on pourrait penser que les Conseils généraux, qui ont des relations privilégiées avec les acteurs historiques du champ (associations et centres communaux d'action sociale), sont rétifs au fait d'autoriser ces nouveaux services d'aide issus du secteur privé lucratif. Mais, dans les faits, les entreprises lucratives ne souhaitent pas être autorisées et leur action syndicale est plutôt orientée vers la disparition du régime de l'autorisation ou son confinement aux activités qui ne sont pas viables économiquement pour le secteur lucratif.

Comme nous l'avons souligné plus haut, le pan d'activité relevant de l'agrément est positionné sur certains segments du champ des services à la personne et représente une part relativement marginale de l'activité d'aide à domicile. La marchandisation au sens stricte de l'aide à domicile est donc somme toute limitée.

\subsection{De la tarification subventionnelle à la tarification marchande : l'expansion de la forme-marchandise par l'évolution de la tarification administrée}

\subsubsection{Le schéma historique de la tarification subventionnelle}

d'activités dont les coûts ne sont pas - ou que partiellement - répercutés sur les usagers. Elles représentent un prix payé pour la réalisation d'un service public. Dans l'aide à domicile, ce subventionnement revêt plusieurs formes. D'une part, il peut s'agir de dotations globales prenant en charge tout ou partie des coûts. Ce mode de financement est relativement marginal et concerne plutôt des projets d'investissement précis ou la mise en place d'expérimentations dans l'aide délivrée aux usagers ${ }^{24}$. D'autre part, le financement peut prendre la forme d'une tarification horaire. Il s'agit aujourd'hui du principal instrument de financement de l'aide à domicile ${ }^{25}$.

La tarification publique, dont il est question ici, doit se distinguer de la régulation tarifaire telle que peut l'exercer la puissance publique dans le cadre de certains secteurs 
(comme les tarifs régulés de l'énergie). Elle se distingue notamment en ce qu'il s'agit d'un tarif de subventionnement, c'est-à-dire que non seulement la puissance publique fixe le tarif mais, de surcroît, elle en assume le coût à travers les différents dispositifs de prise en charge du maintien de l'autonomie financés par les prélèvements obligatoires. Le dispositif de tarification est ainsi l'un des éléments du dispositif de financement de l'aide à domicile, qui repose à la fois sur une logique de solvabilisation de la demande et sur une logique de financement et de structuration de l'offre (que garantit la tarification au coût de revient réel). La tarification doit être appréhendée comme un instrument de subventionnement particulier : le tarif n'est pas un prix marchand, mais une procédure de financement et d'estimation des coûts liés à la production de services collectifs.

C'est la loi de 2002, rénovant l'action sociale et médico-sociale, qui prévoit la prise en charge des coûts de production par le biais d'une tarification, dont la procédure est codifiée dans le Code de l'action sociale et des familles. Si cette tarification est formalisée pour l'aide à domicile dans la loi de 2002, ses principes sont ceux qui ont présidé à la construction de la tarification sociale et médico-sociale dans la seconde moitié du XXe siècle. Cette tarification a longtemps reposé sur trois principes fondamentaux : la prise en charge totale des coûts de production, la reconnaissance des qualifications des travailleurs dans ces coûts de production, et la double opposabilité du tarif (à la fois pour le producteur et pour le financeur du service ${ }^{26}$. La tarification horaire est le fruit d'un double héritage. D'une part, elle découle du principe de tarification qui s'est imposé comme mode de financement dominant dans le secteur médical et médico-social, marqué par une rupture entre le financement public et la production privée de ces services (par les associations notamment). D'autre part, elle est issue du financement de l'aideménagère des caisses de retraite, qui s'est historiquement construit autour d'un barème horaire de participation et donc d'une référence à l'heure d'intervention auprès des usagers.

La tarification subventionnelle est donc en soi un rapport quantitatif, mais elle offre une certaine marge de manœuvre au producteur du service, en ne soumettant pas ses ressources à la validation sociale de la production par l'agrégat de décisions décentralisées. En cela, même si le prix peut être pris en considération, il n'est que l'un des éléments décisionnels dans une relation d'échange fondée plus largement sur un rapport de confiance entre les protagonistes (en l'occurrence entre les associations et organismes publics locaux et les tutelles en charge de l'action sociale et médico-sociale) . L'échange ne se fonde pas sur le prix qui n'est pas décisif dans la validation sociale de l'activité, sa dimension politique et sociale est clairement apparente et ne se dissimule pas derrière le rapport quantitatif du taux d'échange. La tarification subventionnelle procède d'un choix politique d'affecter une part du travail social à une activité menée par un acteur économique (généralement associatif ou public). Dans une démarche de partenariat, qui n'empêche pas les éventuelles tensions et négociations, les financeurs adhèrent à un projet qu'ils financent sur la base de décisions politiques. Il s'agit d'une validation ex ante de l'activité, qui repose sur un choix politique et se rapproche le plus à ce titre de l'activité monétaire non marchande idéale-typique.

\subsubsection{Vers une tarification marchande}

70 Si la tarification des services d'aide à domicile peut être considérée comme une tarification subventionnelle, son évolution récente l'a peu à peu fait glisser vers une tarification de type marchand, en renforçant son rôle paramétrique dans la relation 
d'échange. Deux grandes ruptures tarifaires peuvent être relevées, qui ne se manifestent pas tant du point de vue réglementaire que du point de vue de la pratique des financeurs. Elles remettent en cause les trois grands principes de la tarification subventionnelle jusqu'alors en vigueur (prise en charge totale des coûts de production, reconnaissance des qualifications et double opposabilité du tarif).

71 D'une part, les enveloppes limitatives de crédits sont devenues, dans la pratique, l'un des nouveaux piliers de la tarification des services d'aide à domicile. Ce faisant, une partie des coûts de production peut désormais ne plus être prise en charge par la collectivité publique, remettant en cause l'un des grands principes tarifaires. L'activité des services d'aide ne se trouve ante-validée que dans une certaine limite, ce qui signifie que la validation se déconnecte de l'anticipation de la production.

Cette évolution du dispositif de tarification marque une rupture dans les rapports entre financeurs et producteurs. Le rapport social de la relation d'échange, qui se caractérisait par la négociation et la définition d'objectifs communs, tend à s'effacer derrière une relation totalement intermédiée par le tarif. Il s'agit bien ici, à travers cette réforme, d'une expansion de la rationalité paramétrique caractéristique de la séparation marchande. Cette objectivation par le tarif et le déchirement - ou plutôt la recomposition du rapport social - est un objectif assumé de la refonte du dispositif de tarification. Comme le souligne Hardy, l'esprit du dispositif de tarification a évolué au cours des dernières années vers une rupture avec la gestion tutélaire (ou ce qu'il nomme ici la cogestion).

«Il devenait indispensable de mettre fin à la co-gestion de fait et de ré-internaliser les arbitrages sur les choix de gestion en réintroduisant de la dialectique entre les différents acteurs à l'intérieur des établissements. » (Hardy, 2010, p. 144)

D'autre part, la tarification au coût de revient réel de l'activité, dans le contexte du libre choix de l'usager, constitue une forme de décentralisation de la validation de la production. Les financeurs font le choix de ne plus rembourser a priori les coûts de production de l'aide et acceptent de prendre en charge les coûts de production si et seulement si les usagers ont validé par leur choix les coûts engagés par l'organisme. Le dispositif de tarification des services d'aide à domicile évolue donc d'une tarification subventionnelle à une tarification marchande, puisque le financement à l'activité, conjugué à la non-reprise des déficits, remet en cause le principe de validation collective de la production ex ante, caractéristique de l'activité non marchande. La solvabilisation des usagers, couplée au libre choix du service prestataire, décentralise, décollectivise la décision de validation sociale de la production. En renforçant le rôle paramétrique du prix, l'évolution de la tarification fait pénétrer peu à peu la logique marchande, dans la mesure où le rapport entre les échangistes tend de plus en plus à s'incarner dans un taux d'échange.

Le débat sur l'évolution de la tarification s'accompagne aujourd'hui d'un débat autour de la mise en place d'un référentiel de coûts, sur lequel pourrait reposer une véritable régulation par les prix. L'élaboration de standards, pour une nomenclature des activités de l'aide à domicile (notamment la dichotomie entre l'aide à l'environnement et l'aide à la personne), vise à objectiver les activités et à permettre leur inter-comparaison (AubeMartin et al, 2010 ; Hardy, 2010 ; Poletti, 2012 ; CNSA, 2013).

La logique marchande, reposant sur le principe de séparation et d'objectivation, implique de fait une codification du produit du travail ou du travail lui-même. Marchandisation et codification sont intimement liées, puisque la logique marchande et la rationalité 
paramétrique qui la caractérisent ne peuvent se déployer sans cette codification. Comme le soulignait Marx, pour qu'il ne subsiste plus qu'un rapport quantitatif dans les marchandises, leurs différences qualitatives doivent avoir été supprimées. Pour le dire en reprenant les termes des analyses conventionnalistes, en dehors du marché et préalablement à la relation marchande doivent avoir été construits les dispositifs cognitifs collectifs et les médiations institutionnelles qui permettent aux agents d'interpréter les prix comme les principaux paramètres décisionnels (Eymard-Duvernay, 1989).

De même que la codification des actes et des soins médicaux a accompagné la construction d'un marché de la santé (Batifoulier et al, 2008), la standardisation des activités d'aide à domicile et la volonté d'élaborer des référentiels de coûts autour d'une nomenclature de "paniers de services» constituent les traits saillants de la mise en forme marchandise de l'aide à domicile. La mise en place de ces référentiels doit permettre un recentrage de la relation d'échange sur le prix, devenant alors autant un outil de décision qu'un instrument d'évaluation et de contrôle.

Alors que la tarification subventionnelle s'inscrit plutôt dans une logique de partenariat, où le choix de travailler avec un acteur se fait sur la convergence d'objectifs et de valeurs et où le financement n'intervient qu'ensuite, la tarification de plus en plus fine, fondée sur une codification de l'activité, renverse la logique en faisant du prix l'élément déterminant du partenariat et en ouvrant la voie aux techniques de benchmarking et de scoring. Cette tarification conduit à édulcorer le rapport social dans la relation d'échange en déplaçant le prix du statut de moyen (financement de la production) à celui de fin (maîtrise des dépenses). Il s'agit là d'un glissement de la non-marchandise vers la formemarchandise.

\subsection{Cartographie des activités d'aide à domicile}

Le retour au concept de marchandise offre un outil opérationnel d'analyse du processus de marchandisation, à travers l'étude du rôle paramétrique du prix dans les relations d'échange et de production. Ce cadre analytique nous apparaît d'autant plus adapté au champ de l'aide à domicile qu'on y repère différents cadres tarifaires, où les conventions et les règles de formation des prix délimitent des modèles productifs distincts (Gallois, 2012). Si d'un côté il existe un secteur de l'agrément, marqué par des prix librement administrés par les producteurs et répondant aux principales caractéristiques de la sphère marchande (validation sociale de la production ex post sur la base de décisions individuelles et décentralisées), il existe également un secteur de l'autorisation qui s'est historiquement inscrit dans la sphère non marchande, en étant financé par le biais d'une tarification subventionnelle (validation ex ante de la production sur la base d'une décision collective et centralisée).

79 L'analyse comparative du rôle paramétrique du prix dans ces deux champs distincts permet de dresser une frontière nette entre sphère marchande et non marchande. Cependant, l'évolution récente du dispositif de tarification administrée et son éloignement de l'esprit des textes, dans les pratiques des financeurs, font peu à peu glisser la tarification subventionnelle vers une tarification de type marchande, en renforçant le rôle paramétrique du prix dans la relation d'échange. Aux trois concepts de marchandise, non-marchandise et forme-marchandise correspondent trois formes de prix que l'analyse de l'évolution du champ de l'aide à domicile permet de faire ressortir: le 
prix marchand, la tarification subventionnelle et la tarification marchande. Tandis que le prix marchand fait ressortir le caractère individuel de la transaction, la tarification traduit une implication de la collectivité, mais qui n'est pas du même ordre entre la tarification subventionnelle, qui procède d'une volonté de financement collectif des coûts de production, et la tarification marchande, qui s'apparente plus à une régulation publique tarifaire.

Les activités d'aide à domicile peuvent ainsi être regroupées au sein d'une représentation en forme de quadrants tracés par les axes monétaire et marchand. Tandis que le mouvement historique au $\mathrm{XX}^{\text {ème }}$ siècle a consisté en un déplacement vertical de la production de ces services, du non monétaire vers le monétaire, avec la construction du champ de l'aide à domicile dans le cadre de l'État social et le passage d'un travail familial ou bénévole vers un travail professionnel, les réformes mises en place depuis la fin des années 1990 et le début des années 2000 conduisent à un second déplacement - horizontal cette fois-ci - du non marchand vers le marchand. Mais, ce second déplacement s'opère de deux façons différentes. D'un côté, la stimulation du recours à l'emploi direct dans un premier temps puis le développement du secteur de l'agrément avec le plan Borloo ont accru le domaine de la marchandise. D'un autre côté, et de façon plus massive, l'évolution du dispositif de tarification confère peu à peu à l'activité des services autorisés la forme marchandise. On assiste donc à une marchandisation de l'aide à domicile par deux canaux : d'une part l'hypertrophie - somme toute limitée - de la marchandise et, d'autre part, le glissement de la non-marchandise vers la forme-marchandise.

Formes économiques des activités d'aide à domicile des activités d'aide à domicile en France

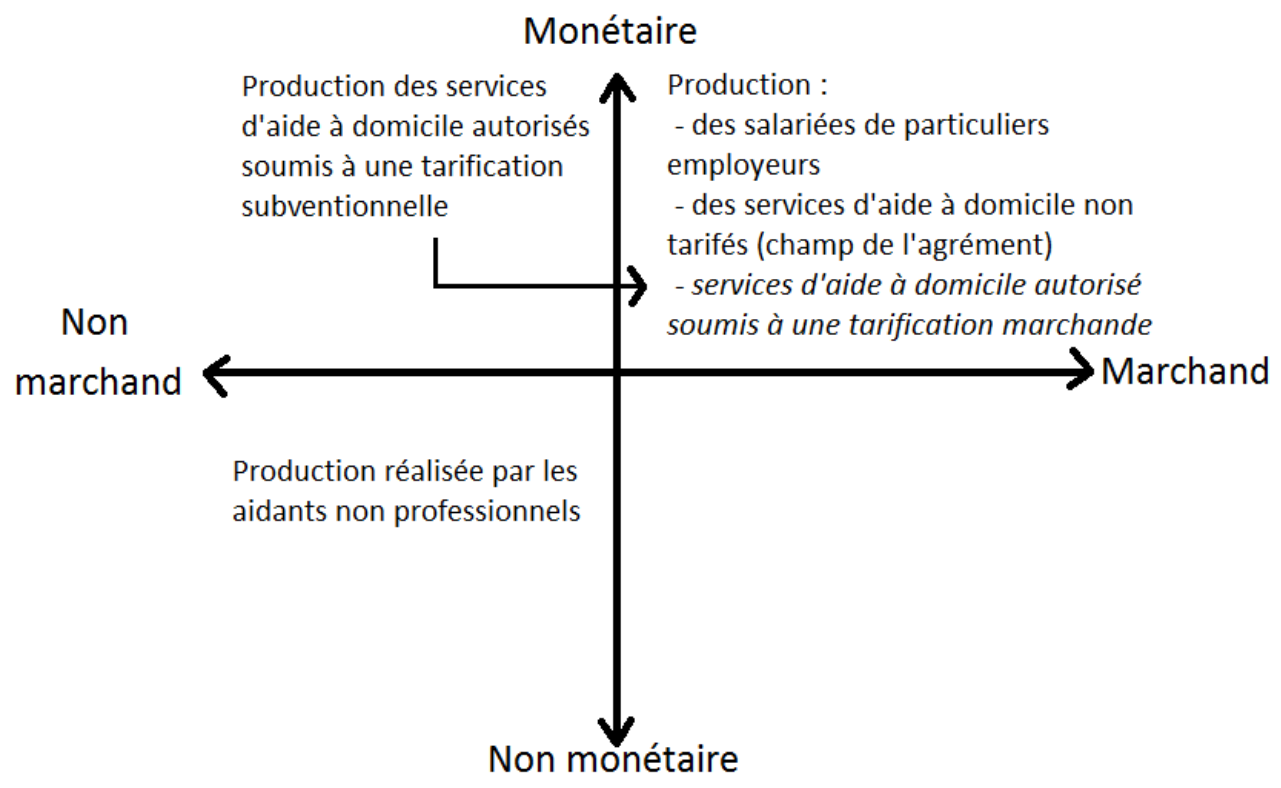

\section{Conclusion}

81 En revenant sur l'analyse théorique du processus de marchandisation, cet article proposait un double objectif. D'une part, il s'agissait de questionner la pertinence de la 
notion de quasi-marché en tant qu'outil conceptuel d'analyse du processus de marchandisation. D'autre part, il s'agissait de proposer un concept alternatif à la notion de marché, arrimé à un cadre théorique plaçant les institutions au cœur de l'analyse économique.

En ciblant notre analyse sur l'évolution des régulations de l'aide à domicile, nous avons ainsi montré que les analyses critiques du processus de marchandisation étaient prisonnières de la notion de marché. Cette notion, dont l'ancrage théorique se situe dans l'analyse économique standard, empêche selon nous ces travaux de prendre la pleine mesure du processus de marchandisation. Au contraire, le retour au concept de marchandise permet de comprendre comment la marchandisation de cette activité se déploie, en dehors même de l'apparition d'organismes à but lucratif et de la mise en concurrence, par le principe de séparation marchande qui réduit le travail d'aide à domicile à un taux objectivé qui médiatise désormais les rapports sociaux.

De notre point de vue, non seulement le recours au concept de marchandise permet de lever le voile sur le principal levier de la marchandisation de l'aide à domicile en France, mais il ouvre en outre la possibilité de déplacer l'analyse de la problématique de la construction sociale des marchés vers celle de la construction sociale de la marchandise. Loin de la seule question de sémantique, il s'agit de porter le regard non plus seulement sur les formes de régulation des échanges mais sur leur articulation avec les formes de régulation de la production. Repartir du concept de marchandise, c'est finalement revenir sur le processus de fracturation entre le mode de régulation des échanges et le mode de régulation de la production, c'est-à-dire offrir un cadre pour l'analyse des transformations du rapport salarial.

\section{BIBLIOGRAPHIE}

Aglietta M., Orléan, A. (1998), La monnaie souveraine, Odile Jacob, Paris.

Aube-Martin, P., Bruant-Bisson A., Reboul (de) J.-B. (2010), Mission relative aux questions de tarification et de solvabilisation des services d'aide à domicile en direction des publics fragiles, Rapport de

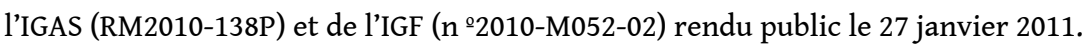

Batifoulier P. (2012), "Le marché de la santé et la reconstruction de l'interaction patientmédecin", Revue Française de Socio-Économie, vol. 2, n 10, pp. 155-174.

Batifoulier P., Domin J.-P., Gadreau M. (2008), "Mutation du patient et construction d'un marché de la santé. L'expérience française", Revue française de socio-économie, vol. 1, n 1, pp. 27-46.

Blanc J. (2007), "Les monnaies sociales : dynamique et logiques des dispositifs", Revue internationale de l'économie sociale - RECMA, $\mathrm{n}^{\circ}$ 303, pp. 30-43.

Bode I. (2007), "La nouvelle donne du marché providence", Retraite Société, vol. 1, n51, pp. 207233.

Branchu C., Jaouen V., Naves, P. (2015), Évaluation des expérimentations relatives à la tarification des services d'aide et d'accompagnement à domicile, Rapport de l'IGAS ( $\left.\mathrm{n}^{\circ} 2014-130 \mathrm{R}\right)$. 
Chantelat P. (2002), "La nouvelle sociologie économique et le lien marchand : des relations personnelles à l'impersonnalité des relations", Revue française de sociologie, vol. 3, n 43, pp. 521556.

CNSA (2011), L'investissement dans les établissements médico-sociaux. L'apport des plans d'aide CNSA. Bilan 2006-2010, CNSA.

CNSA (2013), Cahier des charges pour la réalisation d'une étude des prestations d'aide et d'accompagnement à domicile et des facteurs explicatifs de leurs coûts, Cahier des clauses techniques particulières, version projet du 23 juillet 2013.

Convert B., Heilbron J. (2005), "La réinvention américaine de la sociologie économique", L'Année Sociologique, vol. $55, n^{\circ} 2$, pp. 329-364.

Degavre F., Nyssens M. (2012), Care regimes on the move. Comparing home care for dependent older people in Belgium, England, Germany and Italy, Rapport d'étude pour la Mire.

Devetter F-X. et al (2008), L'aide à domicile face aux services à la personne. Mutations, confusions, paradoxes, Rapport pour la DIIESES.

Devetter F-X., Jany-Catrice F., Ribault T. (2009), Les services à la personne, La Découverte, Paris.

De Vroey M. (1984), "Marchandise, société marchande, société capitaliste. Un réexamen de quelques définitions fondamentales", Cahiers d'économie politique, n 9, pp. 109-135.

Eme B., Laville J.-L. (1999), "Pour une approche pluraliste du tiers secteur", Nouvelles pratiques sociales, vol. $12, \mathrm{n}^{\circ} 1$, pp. 105-125.

England P., Folbre N. (1999), "The Cost of Caring", Annals of the American Academy of Political and Social Science, vol. 561, pp. 39-51.

England P., Folbre, N. (2005), "Gender and Economic Sociology", in: The Handbook of Eonomic Sociology, Princeton University Press and Russel Sage Foundation, Princeton, New York, pp. 627649.

Enjolras B. (1995), Le marché providence : aide à domicile, politique sociale et création d'emploi, Desclée de Brouwer, Paris.

Evers A. (1995), "Part of the welfare mix: The third sector as an intermediate area", Voluntas: International Journal of Voluntary and Nonprofit Organizations, vol. 6, n 2, pp. 159-182.

Eymard-Duvernay F. (1989), "Conventions de qualité et formes de coordination", Revue économique, vol. 40, $\mathrm{n}^{\circ} 2$, pp. 329-360.

Favereau O. (1989), "Marchés internes, marchés externes", Revue économique, vol. 40, n², pp. 273-328.

Folbre N., Nelson J. (2000), "For Love or Money -- Or Both?", The Journal of Economic Perspectives, vol. $14, \mathrm{n}^{\circ} 4$, pp. 123-140.

Fraisse L., Gounouf M.-F. (2008), "Prendre soin de qui ? Les limites de la mise en concurrence des services à la personne", in : L'aide à domicile face aux services à la personne. Mutations, confusions, paradoxes, CLERSE-CRIDA-RESEAU 21, Rapport pour la DIIESES, pp. 223-262.

Fraisse L., Nassaut S. (2010), "La gouvernance par l'évaluation. Les quasi-marchés dans les services à domicile", Annals of Public and Cooperative Economics, vol. 81, n 4, pp. 537-558.

Gallois F. (2012a), Une approche régulationniste des mutations de la configuration institutionnelle française des services à la personne, Thèse de doctorat en Économie, Université de Reims Champagne-Ardennes, Reims, 8 novembre 2012. 
Gallois F. (2012b), « La prise en charge de la dépendance est-elle un problème de santé ou d'emploi ? Une analyse en termes d'espaces de régulation ", Économie et Institutions 1er et 2ème semestre, $\mathrm{n}^{\circ} 18-19$, pp. 35-64.

Gallois F. (2012c), «Le quasi-marché dans les services à la personne : une vision en trois dimensions » dans N. Richez-Battesti, F. Petrella et P. Gianfaldoni Travail, organisations et politiques publiques : quelle "soutenabilité" à l'heure de la mondialisation?, XXXIIèmes Journées de l'Association d'Économie Sociale, Presses Universitaires de Louvain, pp. 335-349.

Gallois F. (2013), "L'organisation publique de la concurrence est-elle source d'efficience ? L'aide à

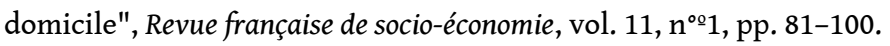

Gallois F., Nieddu M. (2015), "Quand l'État décrète le marché : le cas du Plan Borloo", Revue de la régulation, 1er semestre, $\mathrm{n}^{\circ} 17$.

Gardin L. (2008), "Quelle évolution des modes de régulation ?", in : L'aide à domicile face aux services à la personne. Mutations, confusions, paradoxes, CLERSE-CRIDA-RESEAU 21, Rapport pour la DIIESES, pp. 263-301.

Gardin L., Nyssens M., Minguzzi P. (2010), "Les quasi-marchés dans l'aide à domicile : une mise en perspective européenne", Annals of Public and Cooperative Economics, vol. 81, n 4, pp. 509-536.

Glennerster H., Le Grand J. (1995), "Le développement des quasi-marchés dans la protection sociale", Revue française d'économie, vol. 10, n 3, pp. 111-135.

Gucher C. et al (2011), Non recours et non adhésion : la disjonction des notions de «qualité de vie » et "qualité de l'aide à domicile ", Recherche menée dans le cadre de l'appel à projets CNSA-DREESMiRe autour du thème « Qualité de l'aide au domicile des personnes fragiles », décembre 2008mars 2011

Guerrien B. (2003), "Marchandisation et théorie économique", Actuel Marx, vol. 2, n 34, pp. 121132.

Guerrien B. (2004), La théorie économique néoclassique, 2ème édition, La Découverte, Paris.

Hardy J-P. (2010), Financement et tarification des établissements et services sociaux et médico-sociaux, 3ème ed., Dunod, Paris.

Harribey J-M. (1998), "Marx : des prix de production au circuit monétaire", in : Keynes et Sraffa. Recherche de Passerelles, Cujas, Paris, pp. 157-176.

Harribey J-M. (2008), "Travail, valeur et monnaie : dépoussiérage des catégories marxiennes appliquées à la sphère non marchande", L'Homme et la Société, vol. 2008/4, n 170-171, pp. 127-150.

Harribey J-M. (2009), "Expectation, Financing and Payment of Nonmarket Production: Towards a New Political Economy", International Journal of Political Economy, vol. 38, n 1, pp. 58-80.

Jany-Catrice F. (2010), "La construction sociale du « secteur » des services à la personne : une banalisation programmée ?", Sociologie du travail, vol. 52, n 4, pp. 521-537.

Jany-Catrice F., Vatan S. (2011), "Les pratiques tarifaires des organismes d'aide à domicile de la région Nord-Pas de Calais. Premier panorama", Les synthèses de la DIRECCTE, n 9, novembre 2011.

Kähkönen L. (2004), "Quasi-markets, competition and market failures in local government services", Kommunal ekonomi och politik, vol. 8, n 3, pp. 31-47.

Kähkönen L. (2010), "Competition as a pressure in quasi-markets - Internal inefficiency of an organisation", Public personnel management, vol. 39, n³, pp. 231-242.

Lapidus A. (1986), Le détour de valeur, Economica, Paris. 
Laville J-L. (2000), "Le tiers secteur. Un objet d'étude pour la sociologie économique", Sociologie du travail, vol. $42, \mathrm{n}^{\circ} 4$, pp. 531-550.

Laville J-L. (2003), "Avec Mauss et Polanyi, vers une théorie de l'économie plurielle", Revue du MAUSS, vol. 1, n² 21, pp. 237-249.

Laville J-L. (2008), "Services aux personnes et sociologie économique pluraliste", Revue française de socio-économie, vol. 2, $\mathrm{n}^{\circ}$ 2, pp. 43-58.

Laville J-L., Nyssens M. (2001), Les services sociaux entre associations, Etat et marché, La Découverte et Syros, Paris.

Le Grand J. (1991), "Quasi-markets and Social Policy", The Economic Journal, vol. 101, n 408, pp. $1230-1244$.

Le Grand J. (1999), "Competition, cooperation, or control? Tales from the British National Health Service", Health Affairs (Millwood), vol. 18, n³, pp. 27-39.

Le Grand J., Bartlett W. (1993), Quasi-Markets and Social Policy, Macmillan, Londres.

Marx K. (1859), Critique de l'économie politique, in : Oeuvres, Bibliothèque de La Pléiade [ed. 1965]. Gallimard, Paris, pp. 265-452.

Marx K. (1867.), Le Capital, in : Cuvres, Bibliothèque de La Pléiade [ed. 1965]. Gallimard, Paris, pp. 536-1406.

McMaster R. (2002), "The Analysis of Welfare State Reform: Why the "Quasi-Markets" Narrative Is Descriptively Inadequate and Misleading", . Journal of Economic Issues, vol. 36, n 3, pp. 769-794.

Noguès H. (2008), "Les services d'aide à domicile aux personnes âgées. De la "démarchandisation" à un nouveau marché-providence. Partie 1 : L'émergence des services au sein de l'action sociale", Cahiers documents Cleirppa, $\mathrm{n}^{\circ} 29, \mathrm{pp} .11-14$.

Orléan A. (2003), "Réflexion sur les fondements institutionnels de l'objectivité marchande", Cahiers d'économie politique, vol. 1, nº 44, pp. 181-196.

Orléan A. (2008), "Monnaie, séparation marchande et rapport salarial", in : Conflits et pouvoirs dans les institutions du capitalisme, Presses de Sciences Po, pp. 55-87.

Orléan A., Aglietta M. (2002), La monnaie entre violence et confiance, Odile Jacob, Paris.

Ould Younes S. (2010), "Les services à la personne : une croissance vive en 2007, atténuée en 2008", DARES Analyses, n²0.

Ould Younes S. (2013), "Les services à la personne en 2011 : une baisse globale de l'activité et de l'emploi", DARES Analyses, $\mathrm{n}^{\circ} 25$.

Petrella F. (2012), Aide à domicile et services à la personne : les associations dans la tourmente, Presses Universitaires de Rennes, Rennes.

Petrella F., Richez-Battesti N. (2010), "Régulation de la qualité dans les services à la personne en France : l'Economie sociale et solidaire entre innovation et isomorphisme ?", Management \& Avenir , vol. 35, n 5, 273-292.

Pinville M., Poletti B. (2014), L'évaluation du développement des services à la personne. Rapport d'information de la commission d'évaluation et de contrôle des politiques publiques de l'Assemblée nationale, Paris, La documentation Française.

Polanyi K. (1944), La grande transformation, Gallimard [ed.1983], Paris. 
Poletti B. (2012), Mission relative aux difficultés financières de l'aide à domicile et aux modalités de tarification et d'allocation de ressources des services d'aide à domicile pour publics fragiles. Rapport pour le Ministère des solidarités et de la cohésion sociale.

Postel N., Sobel R. (2010), "Le concept de "marchandise fictive", pierre angulaire de l'institutionnalisme de Karl Polanyi ?", Revue de philosophie économique, vol. 11, n² 2, pp. 3-35.

Poulon F. (2008), Économie générale, 6ème édition, Dunod, Paris.

Rosenbaum E. (2000), "What is a Market? On the Methodology of a Contested Concept", Review of social economy, vol. LVIII, $\mathrm{n}^{\circ}$ 4, pp. 455-482.

Testart A. (2001), "Échange marchand, échange non marchand", Revue française de sociologie, vol. $4, \mathrm{n}^{\circ} 42$, pp. 719-748.

Van de Velde F. (2005), Monnaie, Chômage et capitalisme, Presses Universitaires du Septentrion, Villeneuve d'Ascq.

Vatan S. (2013), "Les mutations de l'aide à domicile en région Nord-Pas-de-Calais, Analyse économique", Les Synthèses de la DIRECCTE, n² 22, mai 2013.

Vatan S. (2014), La tarification des services d'aide à domicile. Une analyse institutionnaliste par le rôle paramétrique du prix, Thèse de doctorat en Économie, Université Lille 1, 8 juillet 2014, Villeneuve d'Ascq.

Williamson O.E. (1973), "Markets and Hierarchies: Some Elementary Considerations", The American Economic Review, vol. 63, nº 2, pp. 316-325.

Zelizer V. (1994), "The Creation of Domestic Currencies", The American Economic Review, vol. 84, $\mathrm{n}$ $\circ 2$, pp. 138-142.

Zelizer V. (2008), "L'économie du care", Revue française de socio-économie, vol. 2, n² 2, pp. 13-25.

Zelizer, V., 2011. Economic Lives: How Culture Shapes the Economy. Princeton University Press, Princeton; Oxford.

\section{NOTES}

1. Nous reprenons ici le concept de théorie standard tel que défini par Favereau (1989).

2. Soit en français respectivement « marchandisation » et "marchéisation ». Notons que, d'une façon générale, le passage de la langue anglaise à la langue française contribue à identifier l'une à l'autre. En effet, la mise en marché est systématiquement assimilée à la marchandisation et la distinction qui existait - au moins dans la sémantique - entre marchéisation et marchandisation disparaît complètement.

3. Insistant sur cette diversité (2007) parle quant à lui de " marchandisation protéiforme ".

4. Si l'on doit le terme de quasi-marché à Williamson, il n'en délivre pourtant aucune définition et cette notion est en réalité remarquablement absente du reste de son œuvre (McMaster, 2002).

5. Dans une démarche assez proche, Kähkönen (2010, p. 231) se réfère également au cadre théorique de l'économie des coûts de transaction en soulignant que « les quasi-marchés n'ont pas de définition exacte, mais qu'ils peuvent être caractérisés comme une forme intermédiaire entre la hiérarchie et le marché conventionnel ». Elle critique alors l'efficacité des quasi-marchés en mettant en avant l'impossibilité de recréer le cadre d'une structure concurrentielle, en pointant notamment la spécificité des services et les asymétries d'information (Kähkönen, 2004, 2010).

6. Si Legrand ne répond pas à la question, Williamson (1973) semble de son côté plutôt ranger les quasi-marchés dans la catégorie des non-marchés. 
7. A côté des Centres communaux d'action sociale (CCAS), les associations sont les acteurs historiques de l'aide à domicile et représentent l'essentiel de l'activité.

8. On appelle reste à charge la partie de l'aide dispensée qui n'est pas financée par les dispositifs de prise en charge du maintien de l'autonomie. Elle incombe donc à l'usager. Ce reste à charge est lié à l'application d'un ticket modérateur et au plafonnement des plans d'aide. L'augmentation du tarif provoque mécaniquement une élévation des restes à charge car le plan d'aide arrive plus vite à saturation.

9. Notons que le « bricolage " par le recours à l'emploi direct mis en place par l'usager pour « rester dans son budget» observé par Gucher et al. dans leurs entretiens ne semblent pas se confirmer au niveau macro-économique. En effet, malgré la forte augmentation de la part des plans d'aide à la charge des bénéficiaires, l'emploi direct, qu'il soit en gré à gré ou en mandataire est en recul depuis plusieurs années.

10. Dans l'aide à domicile, les tutelles financières sont diverses mais nous ferons référence ici principalement aux Conseils départementaux qui sont les chefs de file de l'action sociale et médico-sociales et qui délivrent l'autorisation auprès des organismes d'aide à domicile qui ouvre droit à la tarification administrée.

11. La loi « Hôpital, patients, santé et territoires » voté en 2009 avait pour objectif la réforme du système hospitalier et une refonte de la démographie des établissements de santé. En créant les Agences régionales de santé, elle a très largement affecté le champ social et médico-social.

12. Notons que, pour Laville (2003), l'ancrage théorique de l'économie plurielle se situe dans les travaux de Polanyi mais aussi de Mauss.

13. Cette conception de la monnaie n'est pas propre à Laville. Evers, (1995, p. 163), également défenseur de l'économie plurielle, considère ainsi que " des relations basées sur la monnaie ouvrent un nouvel espace de choix et d'anonymat pour l'individu, mais peuvent également saper des relations sociales fondées sur le lien communautaire ou solidaire ».

14. Dans ces travaux, marchandisation et marchéisation ne sont pas véritablement définis conceptuellement. Précisons également qu'il s'agit d'une représentation structurale des marchés en tant que réseaux sociaux.

15. "The marketization of care could contribute to the costs of care being more widely and equitably distributed, and the provision of care could in some ways be accomplished more effectively." (Folbre et Nelson, 2000, p. 135)

16. Ces quatre formes communautaires constituent l'espace au sein duquel s'est historiquement développée l'aide à domicile non professionnelle.

17. Marx (Marx, 1867, p. 641) utilise le terme de « forme prix » dans son tout premier chapitre du Capital où il revient longuement sur les formes de la valeur. La notion de forme prix qu'il utilise renvoie à la forme monnaie de la valeur, c'est-à-dire à son expression sous la forme de l'équivalent général qu'est la monnaie. Cette forme prix ou forme monnaie découle directement des rapports sociaux qui sont contenus dans la marchandise.

18. Cette idée se retrouve en réalité déjà chez Polanyi : "Il est évident que travail, terre et monnaie ne sont pas des marchandises ; en ce qui les concerne, le postulat selon lequel tout ce qui est acheté et vendu doit avoir été produit pour la vente est carrément faux. En d'autres termes, si l'on s'en tient à la définition empirique de la marchandise, ce ne sont pas des marchandises. » (1944, p. 107). Pour un examen plus en profondeur de la notion de marchandise fictive chez Polanyi et des apports du courant "Critique de l'Économie Politique ", nous renvoyons vers Postel et Sobel (2010).

19. Notons que si Orléan adhère à cette idée de marchandise en tant que rapport social, il le fait en excluant la question des rapports sociaux de production capitalistes. En effet, il analyse le caractère social de la marchandise à partir d'une critique de l'hypothèse de nomenclature, soulignant avec justesse que l'accord collectif permettant la qualification des biens fait de la marchandise une médiation et un rapport social au groupe (Orléan, 2003). A la différence de 
Marx, qui voit dans la marchandise la cristallisation du rapport d'exploitation capitaliste de la force de travail, Orléan, n'adhérant pas à la théorie de la valeur, entrevoit les rapports sociaux de production et l'exploitation capitaliste (ou plutôt la domination de la classe capitaliste) dans l'accès privilégié à la monnaie et à la dette (Orléan, 2008).

20. Notons que si Marx évoque des prix relatifs entre les marchandises lorsqu'il expose les formes de la valeur, il n'y a pas d'ambiguïté sur le fait qu'il n'y ait que des prix monétaires dans la relation marchande dans la mesure où la valeur d'un bien ne s'exprime que dans son rapport à la totalité des marchandises. Seule la monnaie permet de catalyser ce rapport à la totalité marchande, raison pour laquelle le concept de travail abstrait ou travail social qui fonde la théorie de la valeur chez Marx est irrémédiablement liée à la monnaie. Comme le souligne Harribey (Harribey, 1998, p. 160), « il n'y a pas, d'un côté, la valeur en heures de travail, et de l'autre, le prix en monnaie. Sinon, il y aurait une contradiction entre [le fait que] la valeur est la forme revêtue par le travail abstrait et le fait que ce travail abstrait ne se révèle que sur le marché. En effet, sur le marché, il n'y a jamais des heures de travail mais des prix monétaires. »

21. On retrouve l'expression de "rôle paramétrique du prix ", directement liée à la notion de rationalité paramétrique employée par Orléan, sous la plume de Batifoulier, qui fait à juste titre le lien entre l'intensification du rôle paramétrique du prix dans la relation d'échange entre patient et médecin et l'introduction d'une logique marchande : " en reconstruisant un rôle paramétrique du prix, [la rupture progressive avec les principes fondateurs de la Sécurité sociale] conduit les patients à faire des plans en fonction des prix, en phase avec la logique marchande » (Batifoulier, 2012, p. 169).

22. Nous ne nions pas l'importance des coûts dans le processus décisionnel d'anticipation d'une production non marchande. Nous soulignons ici que, la décision étant prise collectivement, la totalité de la production anticipée est validée, dès lors que la production est engagée. Autrement dit, le consentement collectif à payer peut moduler le montant de l'activité qui sera engagé (l'anticipation), mais pas la part qui sera validée (le paiement).

23. Non seulement les Direccte ne disposent pas des moyens suffisants pour effectuer les contrôles mais, de surcroît, le taux maximal d'évolution des prix ne porte que sur la revalorisation annuelle des prix prévus au contrat : « les prix des prestations de service sont librement fixés lors de la signature du contrat conclu entre le prestataire de service et le bénéficiaire. Les prix des prestations contractuelles varient ensuite dans la limite d'un pourcentage fixé par arrêté du ministre chargé de l'économie et des finances compte tenu de l'évolution des salaires et du coût des services " (article L.347-1 du CASF). Il suffit donc aux services d'aide à domicile de faire signer un nouveau contrat aux usagers pour contourner le contrôle des prix.

24. Entre 2006 et 2011, 1,49 milliard d'euros d'investissements ont ainsi été financés par la CNSA pour le développement du champ médico-social dans le cadre des Plans d'aide à l'investissement (CNSA, 2011).

25. Notons que les expérimentations tarifaires en cours, sous l'impulsion de l'Assemblée des départements de France, affichent le recours à la dotation globale. Dans les faits, cette dotation globale est en réalité assise sur une annualisation de la tarification horaire versée par mensualité. Certains compléments tarifaires sont néanmoins versés dans une réelle logique de subventionnant pour la mise en place de nouveaux programmes d'aide.

26. Rappelons que la double opposabilité du tarif signifie que, du côté des producteurs, il est impossible de facturer un dépassement de tarif auprès de l'usager sous peine de retrait de l'autorisation. Du côté du financeur, l'opposabilité du tarif implique qu'il est obligé d'assumer financièrement le coût du service, et notamment les coûts salariaux liés à l'application des conventions collectives. 


\section{RÉSUMÉS}

Ce travail vise à rompre avec l'utilisation de la notion de quasi-marché pour rendre compte du processus de marchandisation des services sociaux en général et de l'aide à domicile en particulier. Nous proposons à travers cette remise en cause d'abandonner toute référence au marché et d'adopter une analyse de la marchandisation à travers le prisme de la marchandise, dont l'ancrage conceptuel se trouve dans la critique de l'économie politique faite par Marx. La marchandisation doit ainsi être comprise comme un processus de mise en marchandise et non seulement de mise en marché. Nous montrons que le retour aux institutions fondamentales de l'économie monétaire de production capitaliste, que sont la monnaie et la marchandise, permet de redonner une cohérence interne aux travaux critiques de l'évolution de la régulation des services sociaux, en les dotant d'un cadre théorique résolument institutionnaliste.

This paper aims at breaking off with the use of the quasi-market notion to analyze the commodification process in social services, and particularly in home care services. We propose to leave any reference to the market by analyzing the commodification process through the concept of commodity, theoretically grounded in the Marx's critique of political economy. Thus, commodification must be understood as a process of commodity constitution rather than a single process of market construction. Finally, we show that a reconnection with the fundamental institutions of monetary production economy, i.e. the money and the commodity, restores the internal consistency of critical researches on the new regulation of social services, by endowing them a theoretical framework firmly institutionalist.

\section{INDEX}

Keywords : quasi-markets, commodification, home care, administrative pricing system Mots-clés : quasi-marchés, marchandisation, aide à domicile, tarification administrée Code JEL H44 - Publicly Provided Goods: Mixed Markets, I11 - Analysis of Health Care Markets, I18 - Government Policy • Regulation • Public Health

\section{AUTEUR}

\section{SYLVAIN VATAN}

Adees Rhône-Alpes, Centre Lillois d'Études et de Recherches Sociologiques et Économiques (CLERSE - UMR 8019 CNRS) Université Lille 1.

sylvain.vatan[at]adeesra.org 